ESAIM: M2AN 50 (2016) 433-454

DOI: $10.1051 / \mathrm{m} 2 \mathrm{an} / 2015052$
ESAIM: Mathematical Modelling and Numerical Analysis

www.esaim-m2an.org

\title{
A NONCONFORMING FINITE ELEMENT APPROXIMATION FOR THE VON KARMAN EQUATIONS
}

\author{
Gouranga Mallik $^{1}$ and NeEla Nataraj ${ }^{1}$
}

\begin{abstract}
In this paper, a nonconforming finite element method has been proposed and analyzed for the von Kármán equations that describe bending of thin elastic plates. Optimal order error estimates in broken energy and $H^{1}$ norms are derived under minimal regularity assumptions. Numerical results that justify the theoretical results are presented.
\end{abstract}

Mathematics Subject Classification. 35J61, 65N12, 65N30.

Received January 29, 2015. Revised May 29, 2015.

Published online February 24, 2016.

\section{INTRODUCTION}

Let $\Omega \subset \mathbb{R}^{2}$ be a polygonal domain with boundary $\partial \Omega$. Consider the von Kármán equations for the deflection of very thin elastic plates that are modeled by a non-linear system of fourth-order partial differential equations with two unknown functions defined by: for given $f \in L^{2}(\Omega)$, seek the vertical displacement $u$ and the Airy stress function $v$ such that

$$
\left.\begin{array}{l}
\Delta^{2} u=[u, v]+f \\
\Delta^{2} v=-\frac{1}{2}[u, u]
\end{array}\right\} \text { in } \Omega
$$

with clamped boundary conditions

$$
u=\frac{\partial u}{\partial \nu}=v=\frac{\partial v}{\partial \nu}=0 \text { on } \partial \Omega,
$$

where the biharmonic operator $\Delta^{2}$ and the von Kármán bracket $[\cdot, \cdot]$ are defined by

$$
\Delta^{2} \varphi:=\varphi_{x x x x}+2 \varphi_{x x y y}+\varphi_{y y y y} \text { and }[\eta, \chi]:=\eta_{x x} \chi_{y y}+\eta_{y y} \chi_{x x}-2 \eta_{x y} \chi_{x y}=\operatorname{cof}\left(D^{2} \eta\right): D^{2} \chi,
$$

$\operatorname{cof}\left(D^{2} \eta\right)$ denotes the co-factor matrix of $D^{2} \eta$ and $\nu$ denotes the unit outward normal to the boundary $\partial \Omega$ of $\Omega$.

Depending on the thickness to length ratio, several plate models have been studied in literature; the most important ones being linear models like Kirchhoff and Reissner-Mindlin plates for thin and moderately thick plates respectively; and non-linear von Kármán plate model for very thin plates. Many practical applications deal with the Kirchhoff model for thin plates in which the transverse shear deformation is negligible. On the other hand, the Reissner-Mindlin plate model for moderately thick plates takes into consideration the shear

\footnotetext{
Keywords and phrases. Von Kármán equations, Morley element, plate bending, non-linear, error estimates.

1 Department of Mathematics, Indian Institute of Technology Bombay Powai, 400076 Mumbai, India.

neela@math.iitb.ac.in; gouranga@math.iitb.ac.in
} 
deformation. The displacements of very thin plates are so large that a non-linear model is essential to consider the membrane action. The assumptions made in the von Kármán model are similar to those of Kirchhoff model except for the linearization of the strain tensor, which in fact, leads to the non-linearity in the model.

For the theoretical study as regards the existence of solutions, regularity and bifurcation phenomena of von Kármán equations, see $[2,4-6,14,19]$ and the references therein. Due to the importance of the problem in application areas, several numerical approaches have also been attempted in the past. The major challenges are posed by the non-linearity and the higher order nature of the equations. The convergence analysis and error bounds for conforming finite element methods are analyzed in [12]. The papers [22,24,25] investigate and analyze the Hellan-Hermann-Miyoshi mixed finite element method and a stress-hybrid method, respectively for the von Kármán equations. In these papers, the authors simultaneously approximate the unknown functions and their derivatives. The papers $[12,22,24]$ deal with the approximation and error bounds for isolated solutions, thereby not discussing the difficulties arising from the non-uniqueness of the solution and the bifurcation phenomena.

Over the last few decades, the finite element methodology has developed in various directions. For higher-order problems, nonconforming methods and discontinuous Galerkin methods are gaining popularity as they have a clear advantage over conforming finite elements with respect to simplicity in implementation. In this paper, an attempt has been made to study the von Kármán equations using nonconforming Morley finite elements. The Morley finite element method has been proposed and analyzed for the biharmonic equation in [21] and for the Monge-Ampère equation in [23]. In [26], a two level additive Schwarz method for a non-linear biharmonic equation using Morley elements is discussed under the assumption of smallness of data. The $C^{0}$ interior penalty method, a variant of the discontinuous Galerkin method has been used to analyze the Monge-Ampère equation in $[9]$.

The solutions $u, v$ of clamped von Kármán equations defined on a polygonal domain belong to $H_{0}^{2}(\Omega) \cap$ $H^{2+\alpha}(\Omega)[6]$, where $\alpha \in\left(\frac{1}{2}, 1\right]$ referred to as the index of elliptic regularity is determined by the interior angles of $\Omega$. Note that when $\Omega$ is convex, $\alpha=1$. This paper discusses a nonconforming finite element discretization of (1.1) - (1.2) and develops a priori error estimates for the displacement and Airy stress functions in polygonal domains with possible corner singularities. To highlight the contributions of this work, we have

- obtained an approximation of an isolated solution pair $(u, v)$ of (1.1) and (1.2) using nonconforming Morley elements;

- developed optimal order error estimates in broken energy and $H^{1}$ norms under realistic regularity assumptions;

- performed numerical experiments that justify the theoretical results.

The advantages of the method are that the nonconforming Morley elements which are based on piecewise quadratic polynomials are simpler to use and have lesser number of degrees of freedom in comparison with the conforming Argyris finite elements with 21 degrees of freedom in a triangle or the Bogner-Fox-Schmit finite elements with 16 degrees of freedom in a rectangle. Moreover, the method is easier to implement than mixed/hybrid finite element methods.

The difficulties due to non-conformity of the space increases the technicalities in the proofs of error estimates. Moreover, one loses the symmetry property with respect to all the variables in the discrete formulation for nonconforming case. An important aid in the proofs is a companion conforming operator, also known in the literature as the enriching operator which maps the elements in the nonconforming finite element space to that of the conforming space. Also, as proved in [17] for the biharmonic problem, it is true that when Morley finite elements are used for the von Kármán equations, the $L^{2}$ error estimates cannot be further improved. This is evident from the results of the numerical experiments presented in Section 5.

The paper is organized as follows. Section 1 is introductory and Section 2 introduces the weak formulation for the problem. This is followed by description of nonconforming finite element formulation in Section 3. Section 4 deals with the existence of the discrete solution and the error estimates in broken energy and $H^{1}$ norms. The results of the numerical experiments are presented in Section 5. Conclusions and perspectives are discussed in Section 6. The analysis of a more generalized form of (1.1) and (1.2) is dealt with in Appendix A. 
Throughout the paper, standard notations on Lebesgue and Sobolev spaces and their norms are employed. We denote the standard $L^{2}$ scalar or vector inner product by $(\cdot, \cdot)$ and the standard norm on $H^{s}(\Omega)$, for $s>0$ by $\|\cdot\|_{s}$. The positive constants $C$ appearing in the inequalities denote generic constants which may depend on the domain $\Omega$ but not on the mesh-size.

\section{WEAK FORMULATION}

The weak formulation corresponding to (1.1), (1.2) is: given $f \in L^{2}(\Omega)$, find $u, v \in V:=H_{0}^{2}(\Omega)$ such that

$$
\begin{aligned}
& a\left(u, \varphi_{1}\right)+b\left(u, v, \varphi_{1}\right)+b\left(v, u, \varphi_{1}\right)=l\left(\varphi_{1}\right) \quad \forall \varphi_{1} \in V \\
& a\left(v, \varphi_{2}\right)-b\left(u, u, \varphi_{2}\right)=0 \quad \forall \varphi_{2} \in V
\end{aligned}
$$

where $\forall \eta, \chi, \varphi \in V$,

$$
a(\eta, \chi):=\int_{\Omega} D^{2} \eta: D^{2} \chi \mathrm{d} x, b(\eta, \chi, \varphi):=\frac{1}{2} \int_{\Omega} \operatorname{cof}\left(D^{2} \eta\right) D \chi \cdot D \varphi \mathrm{d} x \text { and } l(\varphi):=(f, \varphi) .
$$

Note that $b(\cdot, \cdot, \cdot)$ is derived using the divergence-free rows property $[15,23]$. Since the Hessian matrix $D^{2} \eta$ is symmetric, $\operatorname{cof}\left(D^{2} \eta\right)$ is symmetric. Consequently, $b(\cdot, \cdot, \cdot)$ is symmetric with respect to the second and third variables, that is, $b(\eta, \xi, \varphi)=b(\eta, \varphi, \xi)$. Moreover, since $[\cdot, \cdot \cdot]$ is symmetric, $b(\cdot, \cdot, \cdot)$ is symmetric with respect to all the variables in the weak formulation.

An equivalent vector form of the weak formulation which will be also used in the analysis is defined as: for $F=(f, 0)$ with $f \in L^{2}(\Omega)$, seek $\Psi=(u, v) \in \mathscr{V}:=V \times V$ such that

$$
A(\Psi, \Phi)+B(\Psi, \Psi, \Phi)=L(\Phi) \quad \forall \Phi \in \mathscr{V}
$$

where $\forall \Xi=\left(\xi_{1}, \xi_{2}\right), \Theta=\left(\theta_{1}, \theta_{2}\right)$ and $\Phi=\left(\varphi_{1}, \varphi_{2}\right) \in \mathscr{V}$,

$$
\begin{aligned}
& A(\Theta, \Phi):=a\left(\theta_{1}, \varphi_{1}\right)+a\left(\theta_{2}, \varphi_{2}\right), \\
& B(\Xi, \Theta, \Phi):=b\left(\xi_{1}, \theta_{2}, \varphi_{1}\right)+b\left(\xi_{2}, \theta_{1}, \varphi_{1}\right)-b\left(\xi_{1}, \theta_{1}, \varphi_{2}\right) \text { and } \\
& L(\Phi):=\left(f, \varphi_{1}\right) .
\end{aligned}
$$

It is easy to verify that the bilinear forms $A(\cdot, \cdot)$ and $B(\cdot, \cdot, \cdot)$ satisfy the following continuity and coercivity properties. That is, there exist constants $C$ such that

$$
\begin{aligned}
A(\Theta, \Phi) & \leq C\|\Theta\|_{2}\|\Phi\|_{2} \quad \forall \Theta, \Phi \in \mathscr{V}, \\
A(\Theta, \Theta) & \geq C\|\Theta\|_{2}^{2} \quad \forall \Theta \in \mathscr{V}, \\
B(\Xi, \Theta, \Phi) & \leq C\|\Xi\|_{2}\|\Theta\|_{2}\|\Phi\|_{2} \quad \forall \Xi, \Theta, \Phi \in \mathscr{V},
\end{aligned}
$$

where the product norm $\|\Phi\|_{2}:=\sqrt{A(\Phi, \Phi)} \quad \forall \Phi \in \mathscr{V}$. In the sequel, the product norm defined on $\left(H^{s}(\Omega)\right)^{2}$ and $\left(L^{2}(\Omega)\right)^{2}$ are denoted by $\|\cdot\|_{s}$ and $\|\cdot\| \cdot$, respectively.

For the results on existence of solution of the weak formulation, we refer to [2,3,14,19]. More precisely, the weak solution $\Psi=(u, v)$ of $(1.1),(1.2)$ can be characterized as the solution of the operator equation $I \Psi=T \Psi$ defined on $\mathscr{V}$ where $T$ is a compact operator on $\mathscr{V}$ and $I$ is an identity operator on $\mathscr{V}$. In [19], it has been proved that there exists at least one solution of the operator equation. Also, the uniqueness of solution under the assumption on smallness of the data function $f$ has been derived.

In this paper, we follow [12] and assume that the solution $\Psi=(u, v)$ is isolated. That is, the linearized problem defined by: for given $G=\left(g_{1}, g_{2}\right) \in\left(L^{2}(\Omega)\right)^{2} \subset \mathscr{V}^{\prime}$, seek $\Theta=\left(\theta_{1}, \theta_{2}\right) \in \mathscr{V}$ such that

$$
\mathscr{A}(\Theta, \Phi)=(G, \Phi) \quad \forall \Phi \in \mathscr{V}
$$

where $\mathscr{A}(\Theta, \Phi):=A(\Theta, \Phi)+B(\Psi, \Theta, \Phi)+B(\Theta, \Psi, \Phi)$ is well posed and satisfies the a priori bounds

$$
\|\boldsymbol{\Theta}\|_{2} \leq C\|G\|, \quad\|\boldsymbol{\Theta}\|_{2+\alpha} \leq C\|G\|
$$

where $\alpha$ is the index of elliptic regularity. 


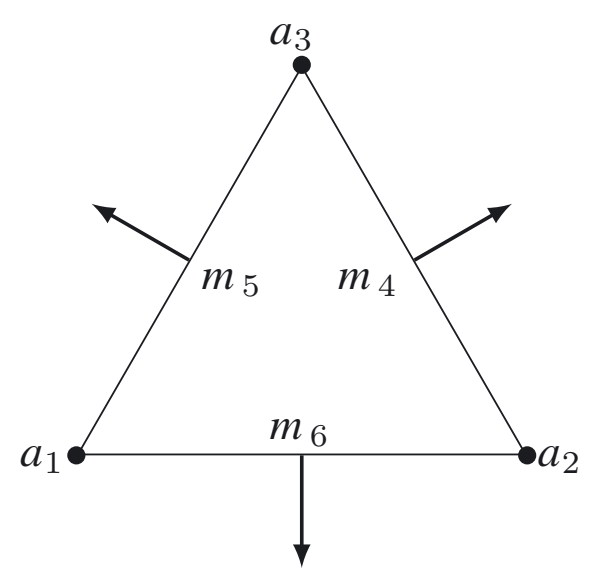

Figure 1. Morley element.

\section{Nonconforming Finite Element Method (NCFEM)}

In the first subsection, the Morley element is defined and some preliminaries are introduced. In the second subsection, nonconforming finite element formulation for von Kármán equations and the corresponding linearized problem are presented. Some properties and auxiliary results necessary for the analysis are discussed in the third subsection.

\subsection{The Morley element}

Let $\mathscr{T}_{h}$ be a regular, quasi-uniform triangulation [10,13] of $\bar{\Omega}$ into closed triangles. Set $h_{T}=\operatorname{diam}(T) \quad \forall T \in$ $\mathscr{T}_{h}$ and $h=\max _{T \in \mathscr{T}_{h}} h_{T}$. For $T \in \mathscr{T}_{h}$ with vertices $a_{i}=\left(x_{i}, y_{i}\right), i=1,2,3$, let $m_{4}, m_{5}$ and $m_{6}$ denote the midpoints of the edges opposite to the vertices $a_{1}, a_{2}$ and $a_{3}$ respectively (see Fig. 1). We denote the set of vertices (resp. edges) of $\mathscr{T}_{h}$ by $\mathfrak{V}_{h}\left(\right.$ resp. $\left.\mathfrak{E}_{h}\right)$. For $e \in \mathfrak{E}_{h}$, let $h_{e}=\operatorname{diam}(e)$.

Definition 3.1. [13] The Morley finite element is a triplet $\left(T, P_{T}, \Phi_{T}\right)$ where

- $T$ is a triangle

- $P_{T}=P_{2}(T)$ is the space of all quadratic polynomials on $T$ and

- $\Phi_{T}=\left\{\phi_{i}\right\}_{i=1}^{6}$ are the degrees of freedom defined by:

$$
\phi_{i}(v)=v\left(a_{i}\right), i=1,2,3 \text { and } \phi_{i}(v)=\frac{\partial v}{\partial \nu}\left(m_{i}\right), i=4,5,6 .
$$

The nonconforming Morley element space associated with the triangulation $\mathscr{T}_{h}$ is defined by

$$
\begin{aligned}
& V_{h}:=\left\{\varphi \in L^{2}(\Omega):\left.\varphi\right|_{T} \in P_{2}(T) \quad \forall T \in \mathscr{T}_{h}, \varphi \text { is continuous at the vertices }\left\{a_{i}\right\}_{i=1}^{3}\right. \text { of the triangle } \\
& \\
& \quad \text { and the normal derivatives of } \varphi \text { at the midpoint of the edges }\left\{m_{i}\right\}_{i=4}^{6} \text { are continuous, } \\
&\left.\varphi=0 \text { at the vertices on } \partial \Omega, \frac{\partial \varphi}{\partial \nu}=0 \text { at the midpoint of the edges on } \partial \Omega\right\} .
\end{aligned}
$$

For $\varphi \in V_{h}$ and $\Phi=\left(\varphi_{1}, \varphi_{2}\right) \in \mathscr{V}_{h}:=V_{h} \times V_{h}$, the mesh dependent semi-norms which are equivalent to the norms denoted as $|\varphi|_{2, h}$ and $\|\Phi\|_{2, h}$, respectively, are defined by:

$$
|\varphi|_{2, h}^{2}:=\sum_{T \in \mathscr{T}_{h}}|\varphi|_{2, T}^{2}, \quad\|\Phi\|_{2, h}^{2}:=\left|\varphi_{1}\right|_{2, h}^{2}+\left|\varphi_{2}\right|_{2, h}^{2} .
$$


Also, for a non-negative integer $m, 1 \leq p<\infty$ and $\varphi \in W^{m, p}\left(\Omega ; \mathscr{T}_{h}\right)$

$$
|\varphi|_{m, p, h}^{2}:=\sum_{T \in \mathscr{T}_{h}}|\varphi|_{m, p, T}^{2}, \quad\|\varphi\|_{m, p, h}^{2}:=\sum_{T \in \mathscr{T}_{h}}\|\varphi\|_{m, p, T}^{2}
$$

and for $p=\infty$

$$
|\varphi|_{m, \infty, h}:=\max _{T \in \mathscr{T}_{h}}|\varphi|_{m, \infty, T}, \quad\|\varphi\|_{m, \infty, h}:=\max _{T \in \mathscr{T}_{h}}\|\varphi\|_{m, \infty, T},
$$

where $|\cdot|_{m, p, T}$ and $\|\cdot\|_{m, p, T}$ denote the usual semi-norm and norm in the Banach space $W^{m, p}(T)$ and $W^{m, p}\left(\Omega ; \mathscr{T}_{h}\right)$ denotes the broken Sobolev space with respect to the mesh $\mathscr{T}_{h}$. For $\Phi=\left(\varphi_{1}, \varphi_{2}\right)$ with $\varphi_{1}, \varphi_{2} \in W^{m, p}\left(\Omega ; \mathscr{T}_{h}\right)$, define $\|\Phi\|_{m, p, h}^{2}:=\left|\varphi_{1}\right|_{m, p, h}^{2}+\left|\varphi_{2}\right|_{m, p, h}^{2}$. When $p=2$, the notation is abbreviated as $|\cdot|_{m, h}$ and $\|\cdot\|_{m, h}$.

\subsection{Nonconforming finite element formulation}

The NCFEM formulation corresponding to (2.1a), (2.1b) can be stated as: for $f \in L^{2}(\Omega)$, seek $\left(u_{h}, v_{h}\right) \in \mathscr{V} / h$ such that

$$
\begin{aligned}
& a_{h}\left(u_{h}, \varphi_{1}\right)+b_{h}\left(u_{h}, v_{h}, \varphi_{1}\right)+b_{h}\left(v_{h}, u_{h}, \varphi_{1}\right)=l_{h}\left(\varphi_{1}\right) \quad \forall \varphi_{1} \in V_{h} \\
& a_{h}\left(v_{h}, \varphi_{2}\right)-b_{h}\left(u_{h}, u_{h}, \varphi_{2}\right)=0 \quad \forall \varphi_{2} \in V_{h}
\end{aligned}
$$

where $\forall \eta, \chi, \varphi \in V_{h}$,

$$
\begin{aligned}
& a_{h}(\eta, \chi):=\sum_{T \in \mathscr{T}_{h}} \int_{T} D^{2} \eta: D^{2} \chi \mathrm{d} x, \quad b_{h}(\eta, \chi, \varphi):=\frac{1}{2} \sum_{T \in \mathscr{T}_{h}} \int_{T} \operatorname{cof}\left(D^{2} \eta\right) D \chi \cdot D \varphi \mathrm{d} x \text { and } \\
& l_{h}(\varphi):=\sum_{T \in \mathscr{T}_{h}} \int_{T} f \varphi \mathrm{d} x .
\end{aligned}
$$

As in the continuous formulation, the discrete form $b_{h}(\cdot, \cdot, \cdot)$ is symmetric with respect to the second and third variables. However, unlike in the conforming case [12], $b_{h}(\cdot, \cdot, \cdot)$ is not symmetric with respect to the first and second variables or the first and third variables. The equivalent vector form corresponding to (3.1a) $-(3.1 \mathrm{~b})$ is given by: seek $\Psi_{h}=\left(u_{h}, v_{h}\right) \in \mathscr{V}_{h}$ such that

$$
A_{h}\left(\Psi_{h}, \Phi\right)+B_{h}\left(\Psi_{h}, \Psi_{h}, \Phi\right)=L_{h}(\Phi) \quad \forall \Phi \in \mathscr{V}_{h}
$$

where $\forall \Xi=\left(\xi_{1}, \xi_{2}\right), \Theta=\left(\theta_{1}, \theta_{2}\right)$ and $\Phi=\left(\varphi_{1}, \varphi_{2}\right) \in \mathscr{V}_{h}$,

$$
\begin{aligned}
& A_{h}(\Theta, \Phi):=a_{h}\left(\theta_{1}, \varphi_{1}\right)+a_{h}\left(\theta_{2}, \varphi_{2}\right), \\
& B_{h}(\Xi, \Theta, \Phi):=b_{h}\left(\xi_{1}, \theta_{2}, \varphi_{1}\right)+b_{h}\left(\xi_{2}, \theta_{1}, \varphi_{1}\right)-b_{h}\left(\xi_{1}, \theta_{1}, \varphi_{2}\right) \text { and } \\
& L_{h}(\Phi):=\sum_{T \in \mathscr{T}_{h}} \int_{T} f \varphi_{1} \mathrm{~d} x .
\end{aligned}
$$

The nonconforming finite element formulation corresponding to (2.9) reads as: for given $G \in\left(L^{2}(\Omega)\right)^{2}$, find $\Theta_{h} \in \mathscr{V}_{h}$ such that

$$
\mathscr{A}_{h}\left(\Theta_{h}, \Phi\right)=(G, \Phi) \quad \forall \Phi \in \mathscr{V}_{h}
$$

where $\mathscr{A}_{h}\left(\Theta_{h}, \Phi\right):=A_{h}\left(\Theta_{h}, \Phi\right)+B_{h}\left(\Psi, \Theta_{h}, \Phi\right)+B_{h}\left(\Theta_{h}, \Psi, \Phi\right)$ and $A_{h}(\cdot, \cdot), B_{h}(\cdot, \cdot, \cdot)$ are defined in (3.3) and (3.4), respectively. 


\subsection{Auxiliary results}

In this subsection, some auxiliary results which are essential for the analysis are stated.

Lemma 3.2 (Integral average [7]). The projection $P_{e}: L^{2}(T) \longrightarrow P_{0}(e)$ defined by $P_{e} \varphi=\frac{1}{h_{e}} \int_{e} \varphi \mathrm{d} s$, satisfies

$$
\left\|\varphi-P_{e} \varphi\right\|_{0, e} \leq C h_{T}^{1 / 2}|\varphi|_{1, T} \quad \forall \varphi \in H^{1}(T)
$$

Lemma 3.3 (Interpolant $[11,13,20])$. Let $\Pi_{h}: V \longrightarrow V_{h}$ be the Morley interpolation operator defined by:

$$
\begin{aligned}
& \left(\Pi_{h} \varphi\right)(p)=\varphi(p) \quad \forall p \in \mathfrak{V}_{h}, \\
& \int_{e} \frac{\partial \Pi_{h} \varphi}{\partial \nu} \mathrm{d} s=\int_{e} \frac{\partial \varphi}{\partial \nu} \mathrm{d} s \quad \forall e \in \mathfrak{E}_{h} .
\end{aligned}
$$

Then for $\varphi \in H^{2+\alpha}(\Omega), \alpha \in(0,1]$, it holds:

$$
\left\|\varphi-\Pi_{h} \varphi\right\|_{m, p, h} \leq C h^{1+\alpha-m+\frac{2}{p}}\|\varphi\|_{2+\alpha}, \quad 0 \leq m \leq 2,1 \leq p<\infty .
$$

For simplicity of notation, the interpolant of $\Phi \in \mathscr{V}$ is denoted by $\Pi_{h} \Phi$ and belongs to $\mathscr{V}_{h}$.

Lemma 3.4 (Enrichment function [11]). Let $V_{c}$ be chosen as Hsieh-Clough-Tocher macro element space [11,13] which is a conforming relative of the Morley finite element space $V_{h}$. For any $\varphi \in V_{h}$, there exists $E_{h} \varphi \in V_{c} \subset V$ such that

$$
\sum_{T \in \mathscr{T}_{h}}\left(h_{T}^{-4}\left|\varphi-E_{h} \varphi\right|_{0, T}^{2}+h_{T}^{-2}\left|\varphi-E_{h} \varphi\right|_{1, T}^{2}\right)+\left|E_{h} \varphi\right|_{2, h}^{2} \leq C|\varphi|_{2, h}^{2}
$$

Again, for $\Phi \in \mathscr{V}_{h}$, the enrichment function corresponding to $\Phi$ denoted by $E_{h} \Phi$, belongs to $\mathscr{V}$.

In the next lemma, we establish an imbedding result. A similar result has been proved in ([26], Lem. 3.1) for the case of convex polygonal domains. However, for the sake of completeness, we provide a detailed proof for the case of polygonal domains. Note that only the edge estimation in (3.12) is different from the proof in [26].

Lemma 3.5 (An imbedding result). For $\varphi \in V_{h}$, it holds:

$$
|\varphi|_{1,4, h} \leq C|\varphi|_{2, h}
$$

Proof. The tangential and normal derivative of $\varphi \in V_{h}$ are continuous at the midpoint of each edges of $T \in \mathscr{T}_{h}$. That is $\varphi_{x}, \varphi_{y} \in S_{h}$ where $S_{h}$ is the nonconforming Crouzeix-Raviart finite element space defined by

$$
\begin{gathered}
S_{h}:=\left\{w \in L^{2}(\Omega):\left.w\right|_{T} \in P_{1}(T) \quad \forall T \in \mathscr{T}_{h}, w\right. \text { is continuous at the midpoints of } \\
\text { the triangle edges and } w=0 \text { at the midpoint of the edges on } \partial \Omega\} .
\end{gathered}
$$

It is enough to prove $|w|_{0,4, h} \leq|w|_{1, h} \quad \forall w \in S_{h}$.

Consider the auxiliary problem: given $\theta \in H^{-1}(\Omega)$, seek $\xi$ such that

$$
-\Delta \xi=\theta \text { in } \Omega, \quad \xi=0 \text { on } \partial \Omega
$$

The solution satisfies the following a priori bounds

$$
\|\xi\|_{1} \leq C\|\theta\|_{-1},\|\xi\|_{1+\gamma} \leq C\|\theta\|
$$


where $\gamma \in\left(\frac{1}{2}, 1\right]$ denotes the elliptic regularity of the problem (3.9). Let $I_{h} \xi \in S_{h}$ be an interpolant which satisfies the estimate $[8,10]$

$$
\left|\xi-I_{h} \xi\right|_{0, h}+h\left|\xi-I_{h} \xi\right|_{1, h} \leq C h^{1+\gamma}\|\xi\|_{1+\gamma} .
$$

A multiplication of (3.9) with $w$ and a use of Green's formula leads to

$$
(\theta, w)=(-\Delta \xi, w)=\sum_{T \in \mathscr{T}_{h}}(\nabla \xi, \nabla w)-\sum_{T \in \mathscr{T}_{h}} \int_{\partial T} \frac{\partial \xi}{\partial \nu} w \mathrm{~d} s
$$

The boundary term can be estimated as follows:

$$
\sum_{T \in \mathscr{T}_{h}} \int_{\partial T} \frac{\partial \xi}{\partial \nu} w \mathrm{~d} s=\sum_{T \in \mathscr{T}_{h}} \sum_{e \subset \partial T} \int_{e} \frac{\partial \xi}{\partial \nu}\left(w-P_{e} w\right) \mathrm{d} s .
$$

Since $\int_{e}\left(w-P_{e} w\right) \mathrm{d} s=0 \forall e \in \mathfrak{E}_{h}$ and $\frac{\partial}{\partial \nu} I_{h} \xi$ is a constant over each edge, we obtain

$$
\begin{aligned}
\sum_{T \in \mathscr{T}_{h}} \sum_{e \subset \partial T} \int_{e} \frac{\partial \xi}{\partial \nu}\left(w-P_{e} w\right) \mathrm{d} s & =\sum_{T \in \mathscr{T}_{h}} \sum_{e \subset \partial T} \int_{e} \frac{\partial}{\partial \nu}\left(\xi-I_{h} \xi\right)\left(w-P_{e} w\right) \mathrm{d} s \\
& \leq \sum_{T \in \mathscr{T}_{h}} \sum_{e \subset \partial T}\left\|\xi-I_{h} \xi\right\|_{1, e}\left\|w-P_{e} w\right\|_{0, e} .
\end{aligned}
$$

A use of trace theorem, Lemma 3.2 and (3.11) leads to the estimate

$$
\left|-\sum_{T \in \mathscr{T}_{h}} \int_{\partial T} \frac{\partial \xi}{\partial \nu} w \mathrm{~d} s\right| \leq C h^{\gamma}\|\xi\|_{1+\gamma}|w|_{1, h} .
$$

Therefore, the a priori bounds in (3.10) yields

$$
(\theta, w) \leq\left(|\xi|_{1}+C h^{\gamma}\|\xi\|_{1+\gamma}\right)|w|_{1, h} \leq C\left(\|\theta\|_{-1}+h^{\gamma}\|\theta\|\right)|w|_{1, h} .
$$

A choice of $\theta=w^{3}$ in (3.14) leads to

$$
|w|_{0,4, h}^{4} \leq C\left(\left\|w^{3}\right\|_{-1}+h^{\gamma}\left\|w^{3}\right\|\right)|w|_{1, h} .
$$

A use of inverse inequality yields

$$
\left\|w^{3}\right\|=\|w\|_{L^{6}(\Omega)}^{3} \leq C h^{-\frac{1}{4}}\|w\|_{L^{4}(\Omega)}^{3} .
$$

Also, Hölder's inequality and the imbedding result $L^{4}(\Omega) \hookrightarrow H_{0}^{1}(\Omega)$ lead to

$$
\left(w^{3}, \xi\right)=\|w\|_{L^{4}(\Omega)}^{3}\|\xi\|_{L^{4}(\Omega)} \leq C\|w\|_{L^{4}(\Omega)}^{3}|\xi|_{1} \Longrightarrow\left\|w^{3}\right\|_{-1} \leq C\|w\|_{L^{4}(\Omega)}^{3} .
$$

Hence, a use of (3.16) and (3.17) in (3.15) leads to the required result

$$
|w|_{0,4, h} \leq C\left(1+h^{\gamma-\frac{1}{4}}\right)|w|_{1, h} \leq C|w|_{1, h} .
$$

The next lemma follows from ([11], Lems. 4.2 and 4.3).

Lemma 3.6 (Bounds for $\left.A_{h}(\cdot, \cdot)\right)$.

(i) Let $\chi \in\left(H^{2+\alpha}(\Omega)\right)^{2}$ and $\Phi \in \mathscr{V}_{h}$. Then, it holds

$$
A_{h}\left(\chi, E_{h} \Phi-\Phi\right) \leq C h^{\alpha}\|\chi\|_{2+\alpha}\|\Phi\|_{2, h} .
$$


(ii) Further, for $\chi \in\left(H^{2+\alpha}(\Omega)\right)^{2}$ and $\Phi \in\left(H_{0}^{2}(\Omega)\right)^{2} \cap\left(H^{2+\alpha}(\Omega)\right)^{2}$, it holds

$$
A_{h}\left(\chi, \Pi_{h} \Phi-\Phi\right) \leq C h^{2 \alpha}\|\chi\|_{2+\alpha}\|\Phi\|_{2+\alpha} .
$$

A use of the definition of $B_{h}(\cdot, \cdot, \cdot)$, generalized Hölder's inequality and Lemma 3.5 leads to a bound given by

$$
B_{h}(\Xi, \Theta, \Phi) \leq C_{b}\|\Xi\|_{2, h}\|\Theta\|_{2, h}\|\Phi\|_{2, h},
$$

where $C_{b}$ is a positive constant independent of $h$.

Lemma 3.7 (A bound for $\left.B_{h}(\cdot, \cdot, \cdot)\right)$. For $\Xi \in\left(H^{2+\alpha}(\Omega)\right)^{2}$ and $\Theta, \Phi \in \mathscr{V}+\mathscr{V}_{h}$, there holds

$$
B_{h}(\Xi, \Theta, \Phi) \leq C\|\Xi\|_{2+\alpha}\|\Theta\|_{1,4, h}\|\Phi\|_{1, h} \leq C\|\Xi\|_{2+\alpha}\|\Theta\|_{2, h}\|\Phi\|_{1, h} .
$$

Proof. Consider

$$
b_{h}(\eta, \chi, \varphi)=\frac{1}{2} \sum_{T \in \mathscr{T}_{h}} \int_{T}\left(\left(\eta_{y y} \chi_{x}-\eta_{x y} \chi_{y}\right) \varphi_{x}+\left(\eta_{x x} \chi_{y}-\eta_{x y} \chi_{x}\right) \varphi_{y}\right) \mathrm{d} x .
$$

For $\eta \in H^{2+\alpha}(\Omega)$, a use of generalized Hölder's inequality and the imbedding result $H^{2+\alpha}(\Omega) \hookrightarrow W^{2,4}(\Omega)$ leads to an estimate of the first term on the right hand side of (3.19) as

$$
\begin{aligned}
\frac{1}{2}\left|\sum_{T \in \mathscr{T}_{h}} \int_{T} \eta_{y y} \chi_{x} \varphi_{x} \mathrm{~d} x\right| & \leq\left(\sum_{T \in \mathscr{T}_{h}}|\eta|_{2,4, T}^{4}\right)^{\frac{1}{4}}\left(\sum_{T \in \mathscr{T}_{h}}|\chi|_{1,4, T}^{4}\right)^{\frac{1}{4}}\left(\sum_{T \in \mathscr{T}_{h}}|\varphi|_{1,2, T}^{2}\right)^{\frac{1}{2}} \\
& \leq\|\eta\|_{W^{2,4}(\Omega)}|\chi|_{1,4, h}|\varphi|_{1, h} \leq C\|\eta\|_{2+\alpha}|\chi|_{1,4, h}|\varphi|_{1, h} .
\end{aligned}
$$

Similar bounds hold true for the remaining three terms in (3.19). Hence the required result follows using the definition of $B_{h}(\cdot, \cdot, \cdot)$ and Lemma 3.5 .

Remark 3.8. Using a proof similar to that of Lemma 3.7, it can be deduced that for $\Xi \in\left(H^{2+\alpha}(\Omega)\right)^{2}$ and $\Theta, \Phi \in \mathscr{V}+\mathscr{V}_{h}$, there holds

$$
B_{h}(\Xi, \Theta, \Phi) \leq C\|\Xi\|_{2+\alpha}\|\Theta\|_{1, h}\|\Phi\|_{1,4, h} \leq C\|\Xi\|_{2+\alpha}\|\Theta\|_{1, h}\|\Phi\|_{2, h} .
$$

Using the definition of $b_{h}(\cdot, \cdot, \cdot)$, an integration by parts and a use of $(3.7)$, the following lemma holds true.

Lemma 3.9 (An intermediate result). For $\eta \in\left(V \cap H^{2+\alpha}(\Omega)\right)+V_{h}$ and $\chi, \varphi \in V_{h}$, it holds

$$
b_{h}(\eta, \chi, \varphi)=b_{h}(\chi, \eta, \varphi)+\frac{1}{2} \sum_{T \in \mathscr{T}_{h}} \int_{\partial T}\left(\eta_{x} \chi_{y}-\eta_{y} \chi_{x}\right) \nabla \varphi \cdot \tau \mathrm{d} s
$$

where $\tau$ is the unit tangent to the boundary $\partial T$ of the triangle T. Moreover,

$$
\forall \eta, \chi, \varphi \in V_{h}, \quad \frac{1}{2} \sum_{T \in \mathscr{T}_{h}} \int_{\partial T}\left(\eta_{x} \chi_{y}-\eta_{y} \chi_{x}\right) \nabla \varphi \cdot \tau \mathrm{d} s \leq C h|\eta|_{2, h}|\chi|_{1, \infty, h}|\varphi|_{2, h} .
$$

Remark 3.10. A use of Lemma 3.9, Remark 3.8 and imbedding result $H^{2+\alpha}(\Omega) \hookrightarrow W^{1, \infty}(\Omega)$ leads to: for $\Xi_{h}, \Phi_{h} \in \mathscr{V}_{h}$ and $\boldsymbol{\xi} \in\left(H^{2+\alpha}(\Omega)\right)^{2}$,

$$
\left|B_{h}\left(\Xi_{h}, \boldsymbol{\xi}, \Phi_{h}\right)\right| \leq\left|B_{h}\left(\boldsymbol{\xi}, \Xi_{h}, \Phi_{h}\right)\right|+C h\left\|\Xi_{h}\right\|_{2, h}\|\boldsymbol{\xi}\|_{2+\alpha}\left\|\Phi_{h}\right\|_{2, h} .
$$


The next lemma which will be used to establish the well posedness of the linearized problem (3.6), follows easily under the assumption that $\Psi$ is an isolated solution of $(2.2)$.

Lemma 3.11 (Well posedness of dual problem). If $\Psi$ is an isolated solution of (2.2), then the dual problem defined by: given $Q \in\left(H^{-1}(\Omega)\right)^{2}$, find $\zeta \in \mathscr{V}$ such that

$$
\mathscr{A}(\Phi, \zeta)=(Q, \Phi) \quad \forall \Phi \in \mathscr{V}
$$

is well posed and satisfies the a priori bounds:

$$
\|\boldsymbol{\zeta}\|_{2} \leq C\|Q\|_{-1}, \quad\|\boldsymbol{\zeta}\|_{2+\alpha} \leq C\|Q\|_{-1}
$$

where $\alpha$ denotes the elliptic regularity index and $\|Q\|_{-1}:=\sup _{\varphi \in\left(H_{0}^{1}(\Omega)\right)^{2}} \frac{(Q, \varphi)}{\|\varphi\|_{1}}$.

Since the Morley finite element space $\mathscr{V}_{h}$ is not a subspace of $\mathscr{V}$ and the discrete form $b_{h}(\cdot, \cdot, \cdot)$ is nonsymmetric with respect to first and second or first and third variables, we encounter additional difficulties in establishing the well posedness of the discrete problem (3.6) in comparison to the conforming case.

Theorem 3.12 (Well posedness of discrete linearized problem). If $\Psi$ is an isolated solution of (2.2), then for sufficiently small $h$, the discrete linearized problem (3.6) is well-posed.

Proof. The space $V_{h}$ being finite dimensional, uniqueness of solution of (3.6) implies existence of solution. Uniqueness follows if an a priori bound for the solution of (3.6) can be established. That is, we aim to prove that

$$
\left\|\Theta_{h}\right\|_{2, h} \leq C\|G\|
$$

for sufficiently small $h$. For $\Phi \in \mathscr{V}_{h}$, using Lemma 3.7 and Remark 3.10, the following Gårding's type inequality holds true:

$$
\begin{aligned}
\mathscr{A}_{h}(\Phi, \Phi) & =A_{h}(\Phi, \Phi)+B_{h}(\Psi, \Phi, \Phi)+B_{h}(\Phi, \Psi, \Phi) \\
& \geq\|\Phi\|_{2, h}^{2}-C\|\Psi\|_{2+\alpha}\|\Phi\|_{2, h}\|\Phi\|_{1, h}-C h\|\Phi\|_{2, h}\|\Psi\|_{2+\alpha}\|\Phi\|_{2, h} .
\end{aligned}
$$

Substitute $\Phi=\Theta_{h}$ in (3.6) and use (3.25) to obtain

$$
\left\|\Theta_{h}\right\|_{2, h} \leq C\left(h\|\Psi\|_{2+\alpha}\left\|\Theta_{h}\right\|_{2, h}+\|\Psi\|_{2+\alpha}\left\|\Theta_{h}\right\|_{1, h}+\|G\|\right) .
$$

Note that

$$
\left\|\Theta_{h}\right\|_{1, h} \leq\left\|\Theta_{h}-E_{h} \Theta_{h}\right\|_{1, h}+\left\|E_{h} \Theta_{h}\right\|_{1, h} \leq C h\left\|\Theta_{h}\right\|_{2, h}+\left\|E_{h} \Theta_{h}\right\|_{1} .
$$

Now we estimate $\left\|E_{h} \Theta_{h}\right\|_{1}$. Choose $Q=-\Delta E_{h} \Theta_{h}$ and $\Phi=E_{h} \Theta_{h}$ in (3.22) and use (3.6) to obtain

$$
\begin{aligned}
& \left\|E_{h} \Theta_{h}\right\|_{1}^{2}=\mathscr{A}\left(E_{h} \Theta_{h}, \boldsymbol{\zeta}\right)=\mathscr{A}_{h}\left(E_{h} \Theta_{h}, \boldsymbol{\zeta}-\Pi_{h} \boldsymbol{\zeta}\right)+\mathscr{A}_{h}\left(E_{h} \Theta_{h}, \Pi_{h} \boldsymbol{\zeta}\right) \\
& =\mathscr{A}_{h}\left(E_{h} \Theta_{h}, \boldsymbol{\zeta}-\Pi_{h} \boldsymbol{\zeta}\right)+\mathscr{A}_{h}\left(E_{h} \Theta_{h}-\Theta_{h}, \Pi_{h} \boldsymbol{\zeta}\right)+\left(G, \Pi_{h} \boldsymbol{\zeta}\right) \\
& =A_{h}\left(E_{h} \Theta_{h}-\Theta_{h}, \boldsymbol{\zeta}\right)+A_{h}\left(\Theta_{h}, \boldsymbol{\zeta}-\Pi_{h} \boldsymbol{\zeta}\right)+B_{h}\left(\Psi, E_{h} \Theta_{h}, \boldsymbol{\zeta}-\Pi_{h} \boldsymbol{\zeta}\right)+B_{h}\left(E_{h} \Theta_{h}, \Psi, \boldsymbol{\zeta}-\Pi_{h} \boldsymbol{\zeta}\right) \\
& +B_{h}\left(\Psi, E_{h} \Theta_{h}-\Theta_{h}, \Pi_{h} \boldsymbol{\zeta}\right)+B_{h}\left(E_{h} \Theta_{h}-\Theta_{h}, \Psi, \Pi_{h} \boldsymbol{\zeta}\right)+\left(G, \Pi_{h} \boldsymbol{\zeta}\right)
\end{aligned}
$$

A use of Lemmas 3.3, 3.4, 3.6, 3.18, 3.23, Remarks 3.8 and 3.10 leads to

$$
\begin{aligned}
\left\|E_{h} \Theta_{h}\right\|_{1}^{2} & \leq C\left(h^{\alpha}\left\|\Theta_{h}\right\|_{2, h}\|\boldsymbol{\zeta}\|_{2+\alpha}+h^{\alpha}\|\Psi\|_{2}\left\|\Theta_{h}\right\|_{2, h}\|\boldsymbol{\zeta}\|_{2+\alpha}+h\|\Psi\|_{2+\alpha}\left\|\Theta_{h}\right\|_{2, h}\|\boldsymbol{\zeta}\|_{2}+\|G\|\|\boldsymbol{\zeta}\|_{2}\right) \\
& \leq C\left(h^{\alpha}\left\|\Theta_{h}\right\|_{2, h}+\|G\|\right)\left\|-\Delta E_{h} \Theta_{h}\right\|_{-1} \leq C\left(h^{\alpha}\left\|\Theta_{h}\right\|_{2, h}+\|G\|\right)\left\|E_{h} \Theta_{h}\right\|_{1} .
\end{aligned}
$$


Therefore,

$$
\left\|E_{h} \Theta_{h}\right\|_{1, h} \leq C\left(h^{\alpha}\left\|\Theta_{h}\right\|_{2, h}+\|G\|\right) .
$$

Now, (3.26)-(3.29) yield

$$
\left\|\Theta_{h}\right\|_{2, h} \leq C_{*} h^{\alpha}\left\|\Theta_{h}\right\|_{2, h}+C\|G\| .
$$

That is, $\left\|\Theta_{h}\right\|_{2, h} \leq C\|G\|$ for a choice of $h \leq h_{1}=\left(\frac{1}{2 C_{*}}\right)^{\frac{1}{\alpha}}$ with $\alpha \in\left(\frac{1}{2}, 1\right]$.

Remark 3.13. If $\Psi$ is an isolated solution of (2.2), then for sufficiently small $h$, the discrete linearized dual problem: given $\mathscr{G} \in\left(L^{2}(\Omega)\right)^{2}$, find $\zeta_{\boldsymbol{h}} \in \mathscr{V}_{h}$ such that

$$
\mathscr{A}_{h}\left(\Phi, \zeta_{h}\right)=(\mathscr{G}, \Phi) \quad \forall \Phi \in \mathscr{V}_{h}
$$

is well posed. The proof is similar to that of Theorem 3.12 and hence is skipped.

\section{Existence, uniqueness And ERror estimates}

In view of Theorem 3.12 and Remark 3.13, the bilinear form $\mathscr{A}_{h}(\cdot, \cdot): \mathscr{V}_{h} \times \mathscr{V}_{h} \rightarrow \mathbb{R}$ defined by

$$
\mathscr{A}_{h}(\Theta, \Phi)=A_{h}(\Theta, \Phi)+B_{h}(\Psi, \Theta, \Phi)+B_{h}(\Theta, \Psi, \Phi)
$$

is nonsingular on $\mathscr{V}_{h} \times \mathscr{V}_{h}$.

The next lemma establishes that the perturbed bilinear form $\tilde{\mathscr{A}}_{h}(\cdot, \cdot)$, constructed using $\Pi_{h} \Psi$ is also nonsingular. Though a similar result is proved in [12] for the conforming case, we provide a proof here for the sake of completeness.

Lemma 4.1 (Nonsingularity of perturbed bilinear form). Let $\Pi_{h} \Psi$ be the interpolation of $\Psi$ as defined in Lemma 3.3. Then, for sufficiently small $h$, the perturbed bilinear form defined by

$$
\tilde{\mathscr{A}}_{h}(\Theta, \Phi)=A_{h}(\Theta, \Phi)+B_{h}\left(\Pi_{h} \Psi, \Theta, \Phi\right)+B_{h}\left(\Theta, \Pi_{h} \Psi, \Phi\right)
$$

is nonsingular on $\mathscr{V}_{h} \times \mathscr{V}_{h}$, if (4.1) is nonsingular on $\mathscr{V}_{h} \times \mathscr{V}_{h}$.

Proof. The bilinear form $\mathscr{A}_{h}: \mathscr{V}_{h} \times \mathscr{V}_{h} \longrightarrow \mathbb{R}$ is bounded and satisfies

$$
\sup _{\|\Theta\|_{2, h}=1} \mathscr{A}_{h}(\Theta, \Phi) \geq \beta\|\Phi\|_{2, h}, \quad \sup _{\|\Phi\|_{2, h}=1} \mathscr{A}_{h}(\Theta, \Phi) \geq \beta\|\Theta\|_{2, h},
$$

where $\beta>0$ is a constant. For $\tilde{\Psi} \in \mathscr{V}+\mathscr{V}_{h}$, a use of the above properties of $\mathscr{A}_{h}(\cdot, \cdot)$ and continuity of $B_{h}(\cdot, \cdot, \cdot)$ (see (3.18)) yields

$$
\begin{aligned}
& \sup _{\|\Phi\|_{2, h}=1} A_{h}(\Theta, \Phi)+B_{h}(\Psi-\tilde{\Psi}, \Theta, \Phi)+B_{h}(\Theta, \Psi-\tilde{\Psi}, \Phi) \\
& \geq \sup _{\|\Phi\|_{2, h}=1} \mathscr{A}_{h}(\Theta, \Phi)-\sup _{\|\Phi\|_{2, h}=1}\left(B_{h}(\tilde{\Psi}, \Theta, \Phi)+B_{h}(\Theta, \tilde{\Psi}, \Phi)\right) \\
& \geq \beta\left\|\Theta_{h}\right\|_{2, h}-2 C_{b}\|\tilde{\Psi}\|_{2, h}\|\Theta\|_{2, h} \geq \frac{\beta}{2}\|\Theta\|_{2, h},
\end{aligned}
$$

provided $\|\tilde{\Psi}\|_{2, h} \leq \frac{\beta}{4 C_{b}}$. Such a choice is justified for sufficiently small $h \leq h_{2}$ (say), by setting $\tilde{\Psi}=\Psi-\Pi_{h} \Psi$ and using Lemma 3.3. Similarly, $\sup _{\|\Theta\|_{2, h}=1} \tilde{\mathscr{A}}_{h}(\Theta, \Phi) \geq \frac{\beta}{2}\|\Phi\|_{2, h} \quad \forall \Phi \in \mathscr{V}_{h}$. Hence the required result. 


\subsection{Existence and local uniqueness results}

Consider the nonlinear operator $\mu: \mathscr{V}_{h} \longrightarrow \mathscr{V}_{h}$ defined by

$$
\tilde{\mathscr{A}}_{h}(\mu(\Theta), \Phi)=L_{h}(\Phi)+B_{h}\left(\Pi_{h} \Psi, \Theta, \Phi\right)+B_{h}\left(\Theta, \Pi_{h} \Psi, \Phi\right)-B_{h}(\Theta, \Theta, \Phi) \quad \forall \Phi \in \mathscr{V}_{h}
$$

A use of Lemma 4.1 leads to the fact that the mapping $\mu$ is well-defined and continuous. Also, any fixed point of $\mu$ is a solution of (3.2) and vice-versa. Hence, in order to show the existence of a solution to (3.2), we will prove that the mapping $\mu$ has a fixed point. As a first step to this, define $\mathbb{B}_{R}\left(\Pi_{h} \Psi\right):=\left\{\Phi \in \mathscr{V}_{h}:\left\|\Phi-\Pi_{h} \Psi\right\|_{2, h} \leq R\right\}$.

Theorem 4.2 (Mapping of ball to ball). For a sufficiently small choice of $h$, there exists a positive constant $R(h)$ such that for any $\Theta \in \mathscr{V}_{h}$,

$$
\left\|\Theta-\Pi_{h} \Psi\right\|_{2, h} \leq R(h) \Rightarrow\left\|\mu(\Theta)-\Pi_{h} \Psi\right\|_{2, h} \leq R(h) .
$$

That is, $\mu$ maps the ball $\mathbb{B}_{R(h)}\left(\Pi_{h} \Psi\right)$ to itself.

Proof. Since the bilinear form $\tilde{\mathscr{A}}_{h}(\cdot, \cdot)$ is nonsingular, from Lemma 4.1, there exists $\bar{\Phi} \in \mathscr{V}_{h}$ such that $\|\bar{\Phi}\|_{2, h}=1$ and

$$
\frac{\beta}{4}\left\|\mu(\Theta)-\Pi_{h} \Psi\right\|_{2, h} \leq \tilde{\mathscr{A}}_{h}\left(\mu(\Theta)-\Pi_{h} \Psi, \bar{\Phi}\right)
$$

Let $E_{h} \bar{\Phi}$ be an enrichment of $\bar{\Phi}$ (see Lem. (3.4)). A use of (4.2), (4.3) and (2.2) yields

$$
\begin{aligned}
\tilde{\mathscr{A}}_{h} & \left(\mu(\Theta)-\Pi_{h} \Psi, \bar{\Phi}\right)=\tilde{\mathscr{A}}_{h}(\mu(\Theta), \bar{\Phi})-\tilde{\mathscr{A}}_{h}\left(\Pi_{h} \Psi, \bar{\Phi}\right) \\
= & L_{h}(\bar{\Phi})+B_{h}\left(\Pi_{h} \Psi, \Theta, \bar{\Phi}\right)+B_{h}\left(\Theta, \Pi_{h} \Psi, \bar{\Phi}\right)-B_{h}(\Theta, \Theta, \bar{\Phi})-A_{h}\left(\Pi_{h} \Psi, \bar{\Phi}\right)-2 B_{h}\left(\Pi_{h} \Psi, \Pi_{h} \Psi, \bar{\Phi}\right) \\
= & L_{h}\left(\bar{\Phi}-E_{h} \bar{\Phi}\right)+\left(A_{h}\left(\Psi, E_{h} \bar{\Phi}\right)-A_{h}\left(\Pi_{h} \Psi, \bar{\Phi}\right)\right)+\left(B_{h}\left(\Psi, \Psi, E_{h} \bar{\Phi}\right)-B_{h}\left(\Pi_{h} \Psi, \Pi_{h} \Psi, \bar{\Phi}\right)\right) \\
& +B_{h}\left(\Pi_{h} \Psi-\Theta, \Theta-\Pi_{h} \Psi, \bar{\Phi}\right)=: T_{1}+T_{2}+T_{3}+T_{4} .
\end{aligned}
$$

Now we estimate $\left\{T_{i}\right\}_{i=1}^{4} . T_{1}$ can be estimated using Lemma 3.4 and the continuity of $L_{h}$. Using Lemma 3.6, continuity of $A_{h}(\cdot, \cdot)$ and Lemma 3.3 , we obtain

$$
T_{2} \leq\left|A_{h}\left(\Psi, E_{h} \bar{\Phi}\right)-A_{h}\left(\Pi_{h} \Psi, \bar{\Phi}\right)\right| \leq\left|A_{h}\left(\Psi, E_{h} \bar{\Phi}-\bar{\Phi}\right)\right|+\left|A_{h}\left(\Psi-\Pi_{h} \Psi, \bar{\Phi}\right)\right| \leq C h^{\alpha}\|\Psi\|_{2+\alpha} .
$$

A use of Lemmas 3.3, 3.4, 3.7 and (3.18) leads to

$$
\begin{aligned}
T_{3} & \leq\left|B_{h}\left(\Psi, \Psi, E_{h} \bar{\Phi}\right)-B_{h}\left(\Pi_{h} \Psi, \Pi_{h} \Psi, \bar{\Phi}\right)\right| \\
& \leq\left|B_{h}\left(\Psi, \Psi, E_{h} \bar{\Phi}-\bar{\Phi}\right)-B_{h}\left(\Pi_{h} \Psi-\Psi, \Pi_{h} \Psi, \Phi\right)-B_{h}\left(\Psi, \Pi_{h} \Psi-\Psi, \bar{\Phi}\right)\right| \\
& \leq C h\|\Psi\|_{2+\alpha}\|\Psi\|_{2}\left\|E_{h} \bar{\Phi}\right\|_{2, h}+C h^{\alpha}\|\Psi\|_{2+\alpha}\|\Psi\|_{2}\|\bar{\Phi}\|_{2, h} \\
& \leq C h^{\alpha}\|\Psi\|_{2}\|\Psi\|_{2+\alpha} .
\end{aligned}
$$

Finally, $T_{4}$ is estimated using (3.18) as

$$
T_{4} \leq\left|B_{h}\left(\Pi_{h} \Psi-\Theta, \Theta-\Pi_{h} \Psi, \bar{\Phi}\right)\right| \leq C\left\|\Theta-\Pi_{h} \Psi\right\|_{2, h}^{2}
$$

A substitution of the estimates derived for $T_{1}, T_{2}, T_{3}$ and $T_{4}$ in (4.4) and an appropriate grouping of the terms yields

$$
\left\|\mu(\Theta)-\Pi_{h} \Psi\right\|_{2, h} \leq C_{1}\left(h^{\alpha}+\left\|\Theta-\Pi_{h} \Psi\right\|_{2, h}^{2}\right)
$$


for some positive constants $C_{1}$ independent of $h$ but dependent on $\|\Psi\|_{2+\alpha}$. A choice of $h \leq h_{3}$, where $h_{3}=$ $\left(\frac{1}{4 C_{1}^{2}}\right)^{\frac{1}{\alpha}}$, yields $4 C_{1}^{2} h^{\alpha} \leq 1$. Since $\left\|\Theta-\Pi_{h} \Psi\right\|_{2, h} \leq R(h)$, for $h \leq h_{3}$, a choice of $R(h):=2 C_{1} h^{\alpha}$ leads to

$$
\left\|\mu(\Theta)-\Pi_{h} \Psi\right\|_{2, h} \leq C_{1} h^{\alpha}\left(1+4 C_{1}^{2} h^{\alpha}\right) \leq R(h)
$$

This completes the proof.

Theorem 4.3 (Existence). For sufficiently small $h$, there exists a solution $\Psi_{h}$ of the discrete problem (3.2) that satisfies $\left\|\Psi_{h}-\Pi_{h} \Psi\right\|_{2, h} \leq R(h)$, for some positive constant $R(h)$ depending on $h$.

Proof. Lemma 4.2 leads to the fact that $\mu$ maps the ball $\mathbb{B}_{R(h)}\left(\Pi_{h} \Psi\right)$ to itself. Therefore, an application of Schauder fixed point theorem [18] yields that the mapping $\mu$ has a fixed point, say $\Psi_{h}$. Hence, $\Psi_{h}$ is an approximate solution of (3.2) which satisfies $\left\|\Psi_{h}-\Pi_{h} \Psi\right\|_{2, h} \leq R(h)$.

Theorem 4.4 (Contraction result). For $\Theta_{1}, \Theta_{2} \in \mathbb{B}_{R(h)}\left(\Pi_{h} \Psi\right)$ with $R(h)$ as defined in Theorem 4.2 , the following contraction result holds true:

$$
\left\|\mu\left(\Theta_{1}\right)-\mu\left(\Theta_{2}\right)\right\|_{2, h} \leq C h^{\alpha}\left\|\Theta_{1}-\Theta_{2}\right\|_{2, h},
$$

for some positive constant $C$ independent of $h$.

Proof. For $\Theta_{1}, \Theta_{2} \in \mathbb{B}_{R(h)}\left(\Pi_{h} \Psi\right)$, let $\mu\left(\Theta_{i}\right), i=1,2$ be the solutions of:

$$
\tilde{\mathscr{A}}_{h}\left(\mu\left(\Theta_{i}\right), \Phi\right)=L_{h}(\Phi)+B_{h}\left(\Pi_{h} \Psi, \Theta_{i}, \Phi\right)+B_{h}\left(\Theta_{i}, \Pi_{h} \Psi, \Phi\right)-B_{h}\left(\Theta_{i}, \Theta_{i}, \Phi\right) \quad \forall \Phi \in \mathscr{V}_{h}
$$

The nonsingularity of $\tilde{\mathscr{A}}_{h}(\cdot, \cdot)$ yields a $\bar{\Phi}$ with $\|\bar{\Phi}\|_{2, h}=1$. With (4.7) and (3.18), we obtain

$$
\begin{aligned}
& \frac{\beta}{4}\left\|\mu\left(\Theta_{1}\right)-\mu\left(\Theta_{2}\right)\right\|_{2, h} \leq \tilde{\mathscr{A}}_{h}\left(\mu\left(\Theta_{1}\right)-\mu\left(\Theta_{2}\right), \bar{\Phi}\right) \\
& =B_{h}\left(\Pi_{h} \Psi, \Theta_{1}-\Theta_{2}, \bar{\Phi}\right)+B_{h}\left(\Theta_{1}-\Theta_{2}, \Pi_{h} \Psi, \bar{\Phi}\right)+B_{h}\left(\Theta_{2}, \Theta_{2}, \bar{\Phi}\right)-B_{h}\left(\Theta_{1}, \Theta_{1}, \bar{\Phi}\right) \\
& =B_{h}\left(\Theta_{2}-\Theta_{1}, \Theta_{1}-\Pi_{h} \Psi, \bar{\Phi}\right)+B_{h}\left(\Theta_{2}-\Pi_{h} \Psi, \Theta_{2}-\Theta_{1}, \bar{\Phi}\right) \\
& \leq C\left\|\Theta_{2}-\Theta_{1}\right\|_{2, h}\left(\left\|\Theta_{1}-\Pi_{h} \Psi\right\|_{2, h}+\left\|\Theta_{2}-\Pi_{h} \Psi\right\|_{2, h}\right) .
\end{aligned}
$$

Since $\Theta_{1}, \Theta_{2} \in \mathbb{B}_{R(h)}\left(\Pi_{h} \Psi\right)$, for a choice of $R(h)$ as in the proof of Theorem 4.2, for sufficiently small $h$, we obtain

$$
\left\|\mu\left(\Theta_{1}\right)-\mu\left(\Theta_{2}\right)\right\|_{2, h} \leq C h^{\alpha}\left\|\Theta_{2}-\Theta_{1}\right\|_{2, h},
$$

for some positive constant $C$ independent of $h$. This completes the proof.

Remark 4.5. (Local uniqueness) Let $\Psi$ be an isolated solution of (2.2). For sufficiently small choice of $h$, Theorem 4.4 establishes the local uniqueness of the solution of (3.2).

\subsection{Error estimates}

In this subsection, the error estimates in the broken energy and $H^{1}$ norms are established.

Theorem 4.6 (Energy norm estimate). Let $\Psi$ and $\Psi_{h}$ be the solutions of (2.2) and (3.2) respectively. Under the assumption that $\Psi$ is an isolated solution, for sufficiently small $h$, it holds

$$
\left\|\Psi-\Psi_{h}\right\|_{2, h} \leq C h^{\alpha},
$$

where $\alpha \in\left(\frac{1}{2}, 1\right]$ is the index of elliptic regularity. 
Proof. A use of triangle inequality yields

$$
\left\|\Psi-\Psi_{h}\right\|_{2, h} \leq\left\|\Psi-\Pi_{h} \Psi\right\|_{2, h}+\left\|\Pi_{h} \Psi-\Psi_{h}\right\|_{2, h} .
$$

For sufficiently small $h$, Theorem 4.3 leads to

$$
\left\|\Pi_{h} \Psi-\Psi_{h}\right\|_{2, h} \leq C h^{\alpha}
$$

Now, Lemma $3.3,(4.11)$ and (4.10) establish the required estimate.

Theorem 4.7 ( $H^{1}$ estimate). Let $\Psi$ and $\Psi_{h}$ be the solutions of (2.2) and (3.2) respectively. Assume that $\Psi$ is an isolated solution. Then, for sufficiently small $h$, it holds

$$
\left\|\Psi-\Psi_{h}\right\|_{1, h} \leq C h^{2 \alpha}
$$

where $\alpha \in\left(\frac{1}{2}, 1\right]$ is the index of elliptic regularity.

Proof. A use of triangle inequality yields

$$
\left\|\Psi-\Psi_{h}\right\|_{1, h} \leq\left\|\Psi-\Pi_{h} \Psi\right\|_{1, h}+\left\|\Pi_{h} \Psi-\Psi_{h}\right\|_{1, h} \leq\left\|\Psi-\Pi_{h} \Psi\right\|_{1, h}+\left\|\boldsymbol{\rho}-E_{h} \boldsymbol{\rho}\right\|_{1, h}+\left\|E_{h} \boldsymbol{\rho}\right\|_{1},
$$

where $\boldsymbol{\rho}=\Pi_{h} \Psi-\Psi_{h}$. A choice of $Q=-\Delta E_{h} \boldsymbol{\rho}$ and $\Phi=E_{h} \boldsymbol{\rho}$ in the dual problem (3.22) and a use of (2.2), (3.2) leads to

$$
\begin{aligned}
\left(\nabla E_{h} \boldsymbol{\rho}, \nabla E_{h} \boldsymbol{\rho}\right)= & \mathscr{A}_{h}\left(E_{h} \boldsymbol{\rho}, \boldsymbol{\zeta}\right)=\mathscr{A}_{h}\left(E_{h} \boldsymbol{\rho}-\boldsymbol{\rho}, \boldsymbol{\zeta}\right)+\mathscr{A}_{h}(\boldsymbol{\rho}, \boldsymbol{\zeta}) \\
= & A_{h}\left(E_{h} \boldsymbol{\rho}-\boldsymbol{\rho}, \boldsymbol{\zeta}\right)+B_{h}\left(\Psi, E_{h} \boldsymbol{\rho}-\boldsymbol{\rho}, \boldsymbol{\zeta}\right)+B_{h}\left(E_{h} \boldsymbol{\rho}-\boldsymbol{\rho}, \Psi, \boldsymbol{\zeta}\right) \\
& +A_{h}\left(\Pi_{h} \Psi-\Psi, \boldsymbol{\zeta}\right)+A_{h}\left(\Psi-\Psi_{h}, \boldsymbol{\zeta}-\Pi_{h} \boldsymbol{\zeta}\right)+A_{h}\left(\Psi, \Pi_{h} \boldsymbol{\zeta}-\boldsymbol{\zeta}\right)+L_{h}\left(\boldsymbol{\zeta}-\Pi_{h} \boldsymbol{\zeta}\right) \\
& +\left(B_{h}\left(\Psi, \Pi_{h} \Psi-\Psi_{h}, \boldsymbol{\zeta}\right)+B_{h}\left(\Pi_{h} \Psi-\Psi_{h}, \Psi, \boldsymbol{\zeta}\right)-B_{h}(\Psi, \Psi, \boldsymbol{\zeta})+B_{h}\left(\Psi_{h}, \Psi_{h}, \Pi_{h} \boldsymbol{\zeta}\right)\right) \\
= & \sum_{i=1}^{8} T_{i} .
\end{aligned}
$$

$T_{1}$ is estimated using Lemma 3.6 and (4.11). $T_{4}$ and $T_{6}$ are estimated using Lemma 3.6. $T_{5}$ is estimated using continuity of $A_{h}(\cdot, \cdot)$, Lemma 3.3 and Theorem 4.6. The term $T_{7}$ is estimated using continuity of $L_{h}$ and Lemma 3.3. $T_{2}$ is estimated using Remark 3.8, Lemma 3.4 and (4.11) as

$$
T_{2} \leq\left|B_{h}\left(\Psi, E_{h} \boldsymbol{\rho}-\boldsymbol{\rho}, \boldsymbol{\zeta}\right)\right| \leq C\|\Psi\|_{2+\alpha}\left\|E_{h} \boldsymbol{\rho}-\boldsymbol{\rho}\right\|_{1, h}\|\boldsymbol{\zeta}\|_{2} \leq C h^{1+\alpha}\|\Psi\|_{2+\alpha}\|\boldsymbol{\zeta}\|_{2} .
$$

$T_{3}$ is estimated using Remark 3.10, Lemma 3.4, (4.15) and (4.11) as

$$
T_{3} \leq\left|B_{h}\left(E_{h} \boldsymbol{\rho}-\boldsymbol{\rho}, \Psi, \boldsymbol{\zeta}\right)\right| \leq\left|B_{h}\left(\Psi, E_{h} \boldsymbol{\rho}-\boldsymbol{\rho}, \boldsymbol{\zeta}\right)\right|+C h\left\|E_{h} \boldsymbol{\rho}-\boldsymbol{\rho}\right\|_{2, h}\|\Psi\|_{2+\alpha}\|\boldsymbol{\zeta}\|_{2} \leq C h^{1+\alpha}\|\Psi\|_{2+\alpha}\|\boldsymbol{\zeta}\|_{2} .
$$

Finally, a use of Remarks 3.8, 3.10, Lemmas 3.3, 3.7, Theorem 4.6 and (3.18) yields an estimate for $T_{8}$ as

$$
\begin{aligned}
T_{8}= & B_{h}\left(\Psi, \Pi_{h} \Psi-\Psi_{h}, \boldsymbol{\zeta}\right)+B_{h}\left(\Pi_{h} \Psi-\Psi_{h}, \Psi, \boldsymbol{\zeta}\right)-B_{h}(\Psi, \Psi, \boldsymbol{\zeta})+B_{h}\left(\Psi_{h}, \Psi_{h}, \Pi_{h} \boldsymbol{\zeta}\right) \\
= & B_{h}\left(\Psi, \Pi_{h} \Psi-\Psi, \boldsymbol{\zeta}\right)+B_{h}\left(\Pi_{h} \Psi-\Psi, \Psi, \boldsymbol{\zeta}\right) \\
& +B_{h}\left(\Psi, \Psi-\Psi_{h}, \boldsymbol{\zeta}\right)+B_{h}\left(\Psi-\Psi_{h}, \Psi, \boldsymbol{\zeta}\right)-B_{h}(\Psi, \Psi, \boldsymbol{\zeta})+B_{h}\left(\Psi_{h}, \Psi_{h}, \Pi_{h} \boldsymbol{\zeta}\right) \\
= & B_{h}\left(\Psi, \Pi_{h} \Psi-\Psi, \boldsymbol{\zeta}\right)+B_{h}\left(\Pi_{h} \Psi-\Psi, \Psi, \boldsymbol{\zeta}\right) \\
& +B_{h}\left(\Psi-\Psi_{h}, \Psi-\Psi_{h}, \boldsymbol{\zeta}\right)+B_{h}\left(\Psi_{h}-\Psi, \Psi_{h}, \Pi_{h} \boldsymbol{\zeta}-\boldsymbol{\zeta}\right)+B_{h}\left(\Psi, \Psi_{h}, \Pi_{h} \boldsymbol{\zeta}-\boldsymbol{\zeta}\right) \\
\leq & C h^{2 \alpha}\left(\|\boldsymbol{\zeta}\|_{2}+\|\boldsymbol{\zeta}\|_{2+\alpha}\right) .
\end{aligned}
$$

A combination of the estimates $T_{1}$ to $T_{8}$ and a priori bounds (3.23) for the linearized dual problem yields

$$
\left(\nabla E_{h} \boldsymbol{\rho}, \nabla E_{h} \boldsymbol{\rho}\right) \leq C h^{2 \alpha}\left\|-\Delta E_{h} \boldsymbol{\rho}\right\|_{-1} \leq C h^{2 \alpha}\left\|E_{h} \boldsymbol{\rho}\right\|_{1} \Longrightarrow\left\|E_{h} \boldsymbol{\rho}\right\|_{1} \leq C h^{2 \alpha} .
$$

A use of Lemmas 3.3, 3.4, (4.11) and the last statement of (4.16) in (4.13) completes the proof. 


\subsection{Convergence of the Newton's method}

In this subsection, we define a working procedure to find an approximation for the discrete solution $\Psi_{h}$. The discrete solution $\Psi_{h}$ of (3.2) is characterized by the fixed point of (4.3). This depends on the unknown $\Pi_{h} \Psi$ and hence the approximate solution for (3.2) is computed using Newton's method in implementation. The iterates of the Newton's method are defined by

$$
A_{h}\left(\Psi_{h}^{n}, \Phi\right)+B_{h}\left(\Psi_{h}^{n-1}, \Psi_{h}^{n}, \Phi\right)+B_{h}\left(\Psi_{h}^{n}, \Psi_{h}^{n-1}, \Phi\right)=B_{h}\left(\Psi_{h}^{n-1}, \Psi_{h}^{n-1}, \Phi\right)+L_{h}(\Phi) \quad \forall \Phi \in \mathscr{V}_{h}
$$

Now we establish that these iterates in fact converge quadratically to the solution of (3.2).

Theorem 4.8 (Convergence of Newton's method). Let $\Psi$ be an isolated solution of (2.2) and let $\Psi_{h}$ solve (3.2). There exists $\rho>0$, independent of $h$, such that for any initial guess $\Psi_{h}^{0}$ which satisfies $\left\|\Psi_{h}^{0}-\Psi_{h}\right\|_{2, h} \leq$ $\rho,\left\|\Psi_{h}^{n}-\Psi_{h}\right\|_{2, h} \leq \frac{\rho}{2^{n}}$ holds true. That is, the iterates of the Newton's method defined in (4.17) are well defined and converge quadratically to $\Psi_{h}$.

Proof. From Lemma 4.1, there exists $\delta>0$ such that for each $Z_{h} \in \mathscr{V}_{h}$ satisfying $\left\|Z_{h}-\Pi_{h} \Psi\right\|_{2, h} \leq \delta$, the form

$$
A_{h}(\Theta, \Phi)+B_{h}\left(Z_{h}, \Theta, \Phi\right)+B_{h}\left(\Theta, Z_{h}, \Phi\right)
$$

is non singular in $\mathscr{V}_{h} \times \mathscr{V}_{h}$. From (4.11), for sufficiently small $h,\left\|\Pi_{h} \Psi-\Psi_{h}\right\|_{2, h} \leq C h^{\alpha}$. Thus $h$ can be chosen sufficiently small so that $\left\|\Pi_{h} \Psi-\Psi_{h}\right\|_{2, h} \leq \frac{\delta}{2}$. Define

$$
\rho:=\min \left\{\frac{\delta}{2}, \frac{\beta}{16 C_{b}}\right\},
$$

where $\beta$ and $C_{b}$ are respectively the coercivity constant of $\mathscr{A}_{h}(\cdot, \cdot)$ and boundedness constant of $B_{h}(\cdot, \cdot, \cdot)$ (see (3.7)). Assume that the initial guess $\Psi_{h}^{0}$ satisfies $\left\|\Psi_{h}-\Psi_{h}^{0}\right\|_{2, h} \leq \rho$. Then

$$
\left\|\Pi_{h} \Psi-\Psi_{h}^{0}\right\|_{2, h} \leq\left\|\Pi_{h} \Psi-\Psi_{h}\right\|_{2, h}+\left\|\Psi_{h}-\Psi_{h}^{0}\right\|_{2, h} \leq \delta
$$

Since (4.18) is nonsingular, the first iterate $\Psi_{h}^{1}$ of the Newton's method in (4.17) is well defined for the initial guess $\Psi_{h}^{0}$. Using the nonsingularity of (4.18), there exists $\bar{\Phi} \in \mathscr{V}_{h}$ such that $\|\bar{\Phi}\|_{2, h}=1$ which satisfies

$$
\frac{\beta}{8}\left\|\Psi_{h}^{1}-\Psi_{h}\right\|_{2, h} \leq A_{h}\left(\Psi_{h}^{1}-\Psi_{h}, \bar{\Phi}\right)+B_{h}\left(\Psi_{h}^{0}, \Psi_{h}^{1}-\Psi_{h}, \bar{\Phi}\right)+B_{h}\left(\Psi_{h}^{1}-\Psi_{h}, \Psi_{h}^{0}, \bar{\Phi}\right) .
$$

A use of (4.17), (3.2), (3.18) yields

$$
\begin{aligned}
& A_{h}\left(\Psi_{h}^{1}-\Psi_{h}, \bar{\Phi}\right)+B_{h}\left(\Psi_{h}^{0}, \Psi_{h}^{1}-\Psi_{h}, \bar{\Phi}\right)+B_{h}\left(\Psi_{h}^{1}-\Psi_{h}, \Psi_{h}^{0}, \bar{\Phi}\right) \\
& =B_{h}\left(\Psi_{h}^{0}, \Psi_{h}^{0}, \bar{\Phi}\right)+L_{h}(\bar{\Phi})-A_{h}\left(\Psi_{h}, \bar{\Phi}\right)-B_{h}\left(\Psi_{h}^{0}, \Psi_{h}, \bar{\Phi}\right)-B_{h}\left(\Psi_{h}, \Psi_{h}^{0}, \bar{\Phi}\right) \\
& =B_{h}\left(\Psi_{h}^{0}, \Psi_{h}^{0}, \bar{\Phi}\right)+B_{h}\left(\Psi_{h}, \Psi_{h}, \bar{\Phi}\right)-B_{h}\left(\Psi_{h}^{0}, \Psi_{h}, \bar{\Phi}\right)-B_{h}\left(\Psi_{h}, \Psi_{h}^{0}, \bar{\Phi}\right) \\
& =B_{h}\left(\Psi_{h}^{0}-\Psi_{h}, \Psi_{h}^{0}-\Psi_{h}, \bar{\Phi}\right) \leq C_{b}\left\|\Psi_{h}^{0}-\Psi_{h}\right\|_{2, h}^{2}
\end{aligned}
$$

Hence, $\left\|\Psi_{h}^{1}-\Psi_{h}\right\|_{2, h} \leq \frac{8 C_{b}}{\beta}\left\|\Psi_{h}^{0}-\Psi_{h}\right\|_{2, h}^{2}$. Since $\left\|\Psi_{h}^{0}-\Psi_{h}\right\| \leq \rho \leq \frac{\beta}{16 C_{b}}$, we obtain

$$
\left\|\Psi_{h}^{1}-\Psi_{h}\right\|_{2, h} \leq \frac{1}{2}\left\|\Psi_{h}^{0}-\Psi_{h}\right\|_{2, h} \leq \frac{\rho}{2} .
$$

Since $\left\|\Psi_{h}^{1}-\Psi_{h}\right\|_{2, h} \leq \rho$, the form (4.18) is nonsingular for $Z_{h}=\Psi_{h}^{1}$. Continuing the process, we obtain

$$
\left\|\Psi_{h}^{n}-\Psi_{h}\right\|_{2, h} \leq \frac{\rho}{2^{n}} .
$$


Moreover, proceeding as in the proof of the estimate (4.20), it can be shown that

$$
\left\|\Psi_{h}^{n+1}-\Psi_{h}\right\|_{2, h} \leq\left(8 C_{b} / \beta\right)\left\|\Psi_{h}^{n}-\Psi_{h}\right\|_{2, h}^{2} .
$$

This establishes that the Newton's method converges quadratically to $\Psi_{h}$. This completes the proof.

Remark 4.9 (local uniqueness). The local uniqueness of solution of (3.2) also follows from Theorem 4.8. We observe that the definition of $\rho$ in (4.19) does not depend on $h$. From Theorem 4.8, it is clear that for any initial guess $\Psi_{h}^{0}$ which lies in the ball of radius $\rho$ with center at $\Psi_{h}$, the sequence generated by (4.17) will converge uniquely to $\Psi_{h}$. In particular, if we choose the initial guess $\Psi_{h}^{0}=\Pi_{h} \Psi$, then the sequence generated by the iterates of the Newton's method will also converge to $\Psi_{h}$ which shows the local uniqueness of the solution $\Psi_{h}$.

\section{NumericAl EXPERIMENTS}

In this section, two numerical experiments that justify the theoretical results are presented. The implementations have been carried out in MATLAB. The results illustrate the order of convergence obtained for the numerical solution of (1.1) and (1.2) computed using the Morley finite element scheme. For a detailed description of construction of basis functions for the Morley element, see Ming and Xu [21]. We implement the Newton's method defined in (4.17) to solve the discrete problem (3.2).

Example 5.1. In the first example, we choose the right hand side load functions such that the exact solution is given by

$$
u(x, y)=x^{2}(1-x)^{2} y^{2}(1-y)^{2} ; \quad v(x, y)=\sin ^{2}(\pi x) \sin ^{2}(\pi y)
$$

on the unit square. The initial triangulation is chosen as shown in Figure 2a. In the uniform red-refinement process, each triangle $T$ is divided into four similar triangles [1] as in Figure $2 \mathrm{~b}$.

Let the mesh parameter at the $N$ th level be denoted by $h_{N}$ and the computational error by $e_{N}$. The experimental order of convergence at the $N$ th level is defined by

$$
\alpha_{N}:=\log \left(e_{N-1} / e_{N}\right) / \log \left(h_{N-1} / h_{N}\right)=\log \left(e_{N-1} / e_{N}\right) / \log (2) .
$$

Tables 1 and 2 show the errors and experimental convergence rates for the variables $u_{h}$ and $v_{h}$. In Figures 3 and 4 , the convergence history of the errors in broken energy, $H^{1}$ and $L^{2}$ norms are illustrated.

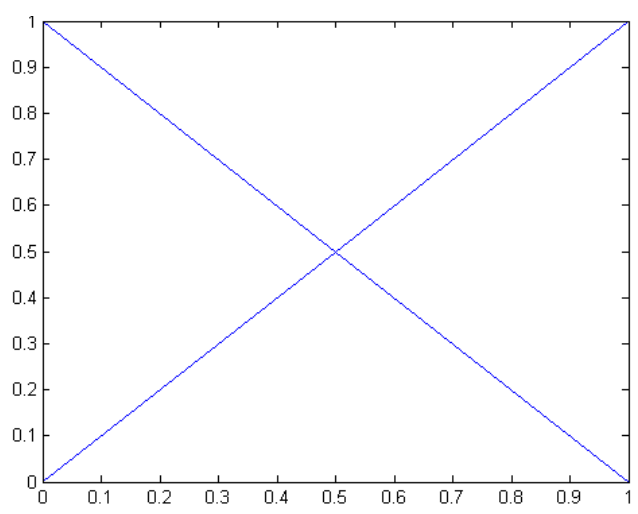

(a)

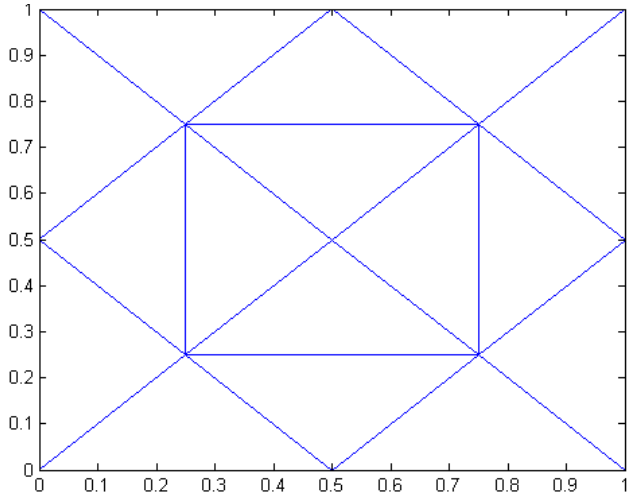

(b)

FIGURE 2. Initial triangulation $\mathscr{T}_{0}$ and its red refinement $\mathscr{T}_{1}$. 
TABLE 1. Errors and convergence rates of $u_{h}$ in broken $H^{2}, H^{1}$ and $L^{2}$ norms.

\begin{tabular}{ccccccc}
\hline \# unknowns & $\left|u-u_{h}\right|_{2, h}$ & Order & $\left|u-u_{h}\right|_{1, h}$ & Order & $\left\|u-u_{h}\right\|_{L^{2}}$ & Order \\
\hline 25 & $0.874685 \mathrm{E}-1$ & - & $0.102155 \mathrm{E}-1$ & - & $0.386068 \mathrm{E}-2$ & - \\
113 & $0.405787 \mathrm{E}-1$ & 1.1080 & $0.257318 \mathrm{E}-2$ & 1.9891 & $0.919743 \mathrm{E}-3$ & 2.0695 \\
481 & $0.209921 \mathrm{E}-1$ & 0.9508 & $0.732470 \mathrm{E}-3$ & 1.8127 & $0.248134 \mathrm{E}-3$ & 1.8901 \\
1985 & $0.106209 \mathrm{E}-1$ & 0.9829 & $0.191118 \mathrm{E}-3$ & 1.9383 & $0.636227 \mathrm{E}-4$ & 1.9635 \\
8065 & $0.532754 \mathrm{E}-2$ & 0.9953 & $0.483404 \mathrm{E}-4$ & 1.9831 & $0.160158 \mathrm{E}-4$ & 1.9900 \\
32513 & $0.266595 \mathrm{E}-2$ & 0.9988 & $0.121213 \mathrm{E}-4$ & 1.9956 & $0.401107 \mathrm{E}-5$ & 1.9974 \\
\hline
\end{tabular}

TABLE 2. Errors and convergence rates of $v_{h}$ in broken $H^{2}, H^{1}$ and $L^{2}$ norms.

\begin{tabular}{ccccccc}
\hline \# unknowns & $\left|v-v_{h}\right|_{2, h}$ & Order & $\left|v-v_{h}\right|_{1, h}$ & Order & $\left\|v-v_{h}\right\|_{L^{2}}$ & Order \\
\hline 25 & 19.245671 & - & $2.140613 \mathrm{E}-0$ & - & $0.770876 \mathrm{E}-0$ & - \\
113 & 9.5043699 & 1.0178 & $0.569979 \mathrm{E}-0$ & 1.9090 & $0.177898 \mathrm{E}-0$ & 2.1154 \\
481 & 5.0549209 & 0.9109 & $0.161737 \mathrm{E}-0$ & 1.8172 & $0.482777 \mathrm{E}-1$ & 1.8816 \\
1985 & 2.5758939 & 0.9726 & $0.421546 \mathrm{E}-1$ & 1.9398 & $0.123930 \mathrm{E}-1$ & 1.9618 \\
8065 & 1.2944929 & 0.9926 & $0.106618 \mathrm{E}-1$ & 1.9832 & $0.312076 \mathrm{E}-2$ & 1.9895 \\
32513 & 0.6480848 & 0.9981 & $0.267351 \mathrm{E}-2$ & 1.9956 & $0.781643 \mathrm{E}-3$ & 1.9973 \\
\hline
\end{tabular}

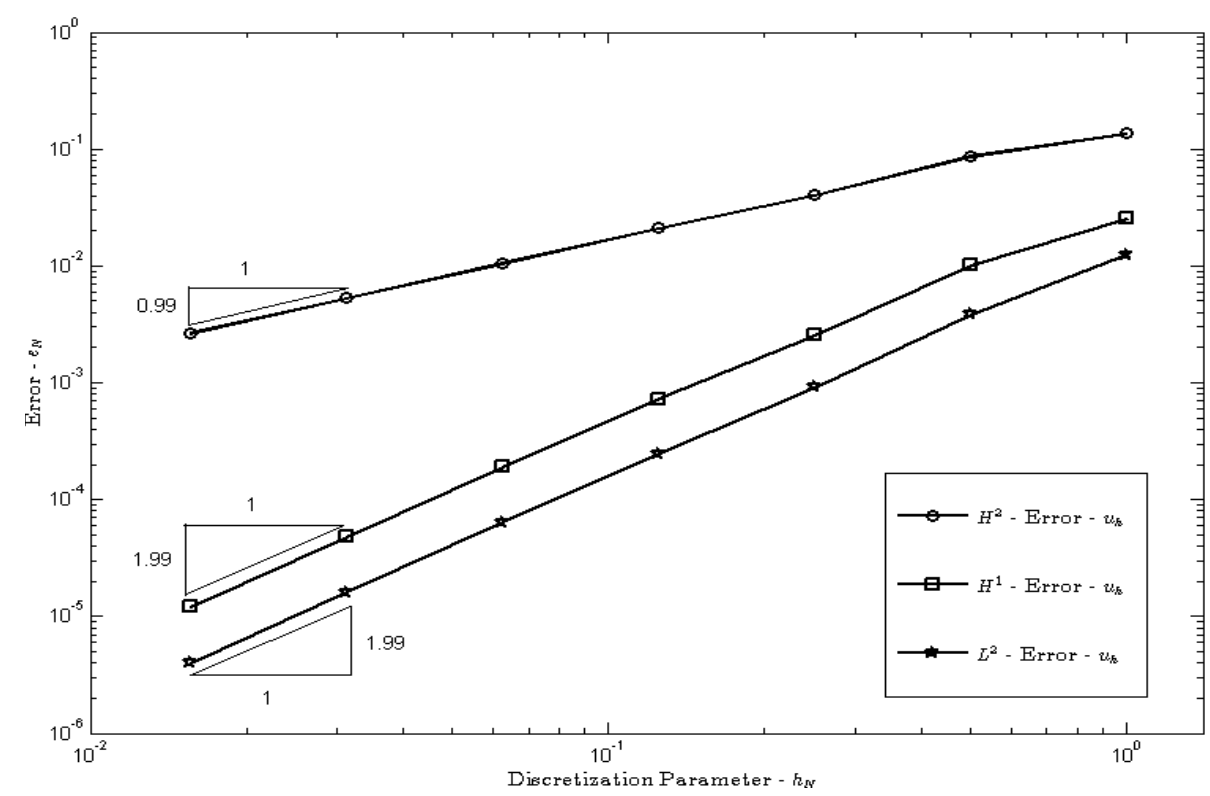

FiguRE 3. Convergence history of displacement for Example 1.

The computational order of convergences in broken $H^{2}, H^{1}$ norms are quasi-optimal and verify the theoretical results obtained in Theorems 4.6 and 4.7 for $\alpha=1$. The order of convergence with respect to $L^{2}$ norm is sub-optimal justifying the results in [17] that using a lower order finite element method, the order of convergence in $L^{2}$ norm cannot be improved than that of the $H^{1}$ norm. 


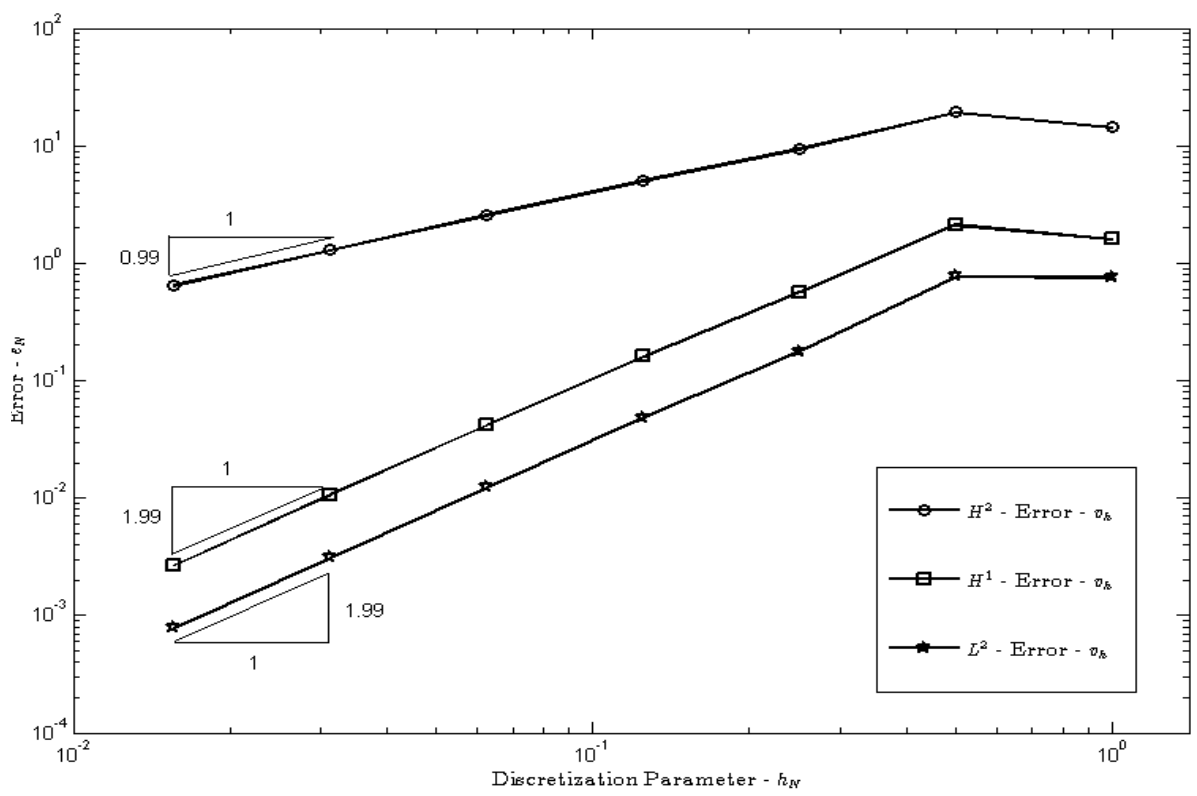

FiguRE 4. Convergence history of Airy stress for Example 1.

TABLE 3. Errors and the experimental convergence rates for $u_{h}$ in broken $H^{2}, H^{1}$ and $L^{2}$ norms for $L$-shaped domain.

\begin{tabular}{ccccccc}
\hline \# unknowns & $\left|u-u_{h}\right|_{2, h}$ & Order & $\left|u-u_{h}\right|_{1, h}$ & Order & $\left\|u-u_{h}\right\|_{L^{2}}$ & Order \\
\hline 17 & 29.209171 & - & $6.363539 \mathrm{E}-0$ & - & $2.769499 \mathrm{E}-0$ & - \\
81 & 14.130192 & 1.0476 & $1.682747 \mathrm{E}-0$ & 1.9190 & $0.693436 \mathrm{E}-0$ & 1.9977 \\
353 & 7.5651300 & 0.9013 & $0.491659 \mathrm{E}-0$ & 1.7750 & $0.200814 \mathrm{E}-0$ & 1.7879 \\
1473 & 3.9620126 & 0.9331 & $0.146551 \mathrm{E}-0$ & 1.7462 & $0.583024 \mathrm{E}-1$ & 1.7842 \\
6017 & 2.0841141 & 0.9267 & $0.487106 \mathrm{E}-1$ & 1.5891 & $0.179703 \mathrm{E}-1$ & 1.6979 \\
24321 & 1.1252534 & 0.8891 & $0.187772 \mathrm{E}-1$ & 1.3752 & $0.613474 \mathrm{E}-2$ & 1.5505 \\
\hline
\end{tabular}

Example 5.2. Consider the $L$-shaped domain $\Omega=(-1,1)^{2} \backslash([0,1) \times(-1,0])$ (see Fig. 5). Choose the right hand functions such that the exact singular solution [16] in polar coordinates is given by

$$
u(r, \theta)=\left(r^{2} \cos ^{2} \theta-1\right)^{2}\left(r^{2} \sin ^{2} \theta-1\right)^{2} r^{1+\alpha} g_{\alpha, \omega}(\theta) ; \quad v(r, \theta)=u(r, \theta),
$$

where $\omega:=\frac{3 \pi}{2}$ and $\alpha:=0.5444837367$ is a non-characteristic root of $\sin ^{2}(\alpha \omega)=\alpha^{2} \sin ^{2}(\omega)$ with

$$
\begin{aligned}
g_{\alpha, \omega}(\theta)= & \left(\frac{1}{\alpha-1} \sin ((\alpha-1) \omega)-\frac{1}{\alpha+1} \sin ((\alpha+1) \omega)\right)(\cos ((\alpha-1) \theta)-\cos ((\alpha+1) \theta)) \\
& -\left(\frac{1}{\alpha-1} \sin ((\alpha-1) \theta)-\frac{1}{\alpha+1} \sin ((\alpha+1) \theta)\right)(\cos ((\alpha-1) \omega)-\cos ((\alpha+1) \omega)) .
\end{aligned}
$$

Tables 3 and 4 show the errors and experimental convergence rates for the variables $u_{h}$ and $v_{h}$. The domain being non-convex, we do not obtain linear and quadratic order of convergences in broken energy and $H^{1}$ norms for displacement and Airy stress functions. 

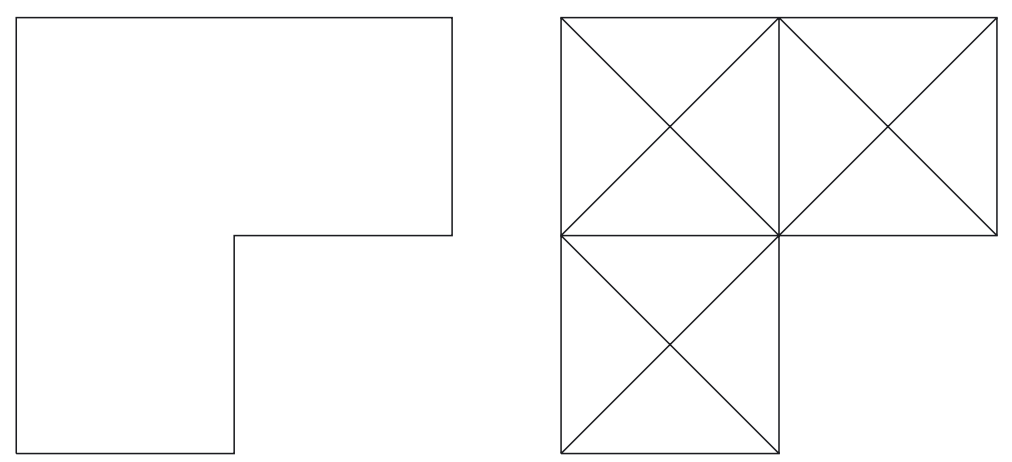

FiguRE 5. $L$-shaped domain and its initial triangulation.

TABLE 4. Errors and the experimental convergence rates for $v_{h}$ in broken $H^{2}, H^{1}$ and $L^{2}$ norms for $L$-shaped domain.

\begin{tabular}{ccccccc}
\hline \# unknowns & $\left|v-v_{h}\right|_{2, h}$ & Order & $\left|v-v_{h}\right|_{1, h}$ & Order & $\left\|v-v_{h}\right\|_{L^{2}}$ & Order \\
\hline 17 & 24.759835 & - & $4.932699 \mathrm{E}-0$ & - & $2.069151 \mathrm{E}-0$ & - \\
81 & 15.293270 & 0.6951 & $1.779132 \mathrm{E}-0$ & 1.4712 & $0.727981 \mathrm{E}-0$ & 1.5070 \\
353 & 7.8509322 & 0.9619 & $0.483823 \mathrm{E}-0$ & 1.8786 & $0.199644 \mathrm{E}-0$ & 1.8664 \\
1473 & 4.0531269 & 0.9538 & $0.137278 \mathrm{E}-0$ & 1.8173 & $0.557622 \mathrm{E}-1$ & 1.8400 \\
6017 & 2.1219988 & 0.9336 & $0.439086 \mathrm{E}-1$ & 1.6445 & $0.165699 \mathrm{E}-1$ & 1.7507 \\
24321 & 1.1421938 & 0.8936 & $0.165883 \mathrm{E}-1$ & 1.4043 & $0.545066 \mathrm{E}-2$ & 1.6040 \\
\hline
\end{tabular}

\section{Conclusions And Perspectives}

In this work, an attempt has been made to obtain approximate solutions for the clamped von Kármán equations defined on polygonal domains using nonconforming Morley elements. Error estimates in broken energy and $H^{1}$ norms are established for sufficiently small discretization parameters. Numerical results that substantiate the theoretical results are obtained. A future area of interest would be derivation of reliable a posteriori error estimates that drive the adaptive mesh refinements.

\section{Appendix A.}

We consider one of the variants of von Kármán equations which is important in practical applications and give a brief sketch of the extension of the analysis. Consider the following form of von Kármán equations:

$$
\left.\begin{array}{l}
\Delta^{2} u=[u, v]-\frac{p}{D} \Delta u+f \\
\Delta^{2} v=-\frac{1}{2}[u, u]
\end{array}\right\} \text { in } \Omega
$$

with clamped boundary conditions

$$
u=\frac{\partial u}{\partial \nu}=v=\frac{\partial v}{\partial \nu}=0 \text { on } \partial \Omega,
$$

where $p$ is a real parameter known as the bifurcation parameter and $D$ denotes the flexural rigidity of the plate. The weak formulation of (A.1)-(A.2) reads as: given $F=(f, 0)$, find $\Psi \in \mathscr{V}$ such that

$$
A(\Psi, \Phi)+B(\Psi, \Psi, \Phi)+\mathfrak{C}(\Psi, \Phi)=L(\Phi) \quad \forall \Phi \in \mathscr{V}
$$


where $A(\cdot, \cdot), B(\cdot, \cdot, \cdot), L(\cdot)$ are defined in $(2.3)-(2.5)$ respectively, and $\mathfrak{C}(\cdot, \cdot)$ is defined as

$$
\mathfrak{C}(\Theta, \Phi)=-\frac{p}{D} \int_{\Omega} \nabla \theta_{1} \cdot \nabla \varphi_{1} \mathrm{~d} x \quad \forall \Theta=\left(\theta_{1}, \theta_{2}\right) \text { and } \Phi=\left(\varphi_{1}, \varphi_{2}\right) \in \mathscr{V}
$$

The corresponding nonconforming finite element formulation is given by: find $\Psi_{h} \in \mathscr{V}_{h}$ such that

$$
A_{h}\left(\Psi_{h}, \Phi\right)+B_{h}\left(\Psi_{h}, \Psi_{h}, \Phi\right)+\mathfrak{C}_{h}\left(\Psi_{h}, \Phi\right)=L_{h}(\Phi) \quad \forall \Phi \in \mathscr{V}_{h}
$$

where $A_{h}(\cdot, \cdot), B_{h}(\cdot, \cdot, \cdot), L_{h}(\cdot)$ are defined in $(3.3)-(3.5)$ respectively, and $\mathfrak{C}_{h}(\cdot, \cdot)$ is defined as

$$
\mathfrak{C}_{h}(\Theta, \Phi)=-\frac{p}{D} \sum_{T \in \mathscr{T}_{h}} \int_{T} \nabla \theta_{1} \cdot \nabla \varphi_{1} \mathrm{~d} x \quad \forall \Theta=\left(\theta_{1}, \theta_{2}\right) \text { and } \Phi=\left(\varphi_{1}, \varphi_{2}\right) \in \mathscr{V}_{h} .
$$

For the newly introduced bilinear form $\mathfrak{C}(\cdot, \cdot)$, the following boundedness properties hold true:

$$
\begin{aligned}
\mathfrak{C}(\Theta, \Phi) & \leq C\|\Theta\|_{1}\|\Phi\|_{1} \quad \forall \Theta, \Phi \in \mathscr{V} \\
\mathfrak{C}_{h}\left(\Theta_{h}, \Phi_{h}\right) & \leq C\left\|\Theta_{h}\right\|_{1, h}\left\|\Phi_{h}\right\|_{1, h} \quad \forall \Theta_{h}, \Phi_{h} \in \mathscr{V}_{h} .
\end{aligned}
$$

For the modified problem (A.3), the linearized problem (see (3.6)) is defined by: for given $G \in\left(L^{2}(\Omega)\right)^{2}$, find $\Theta \in \mathscr{V}$ such that

$$
\mathscr{A}(\Theta, \Phi)=(G, \Phi) \quad \forall \Phi \in \mathscr{V}
$$

where

$$
\mathscr{A}(\Theta, \Phi):=A(\Theta, \Phi)+B(\Psi, \Theta, \Phi)+B(\Theta, \Psi, \Phi)+\mathfrak{C}(\Theta, \Psi) .
$$

The dual problem is stated as: given $Q \in\left(H^{-1}(\Omega)\right)^{2}$, find $\zeta \in \mathscr{V}$ such that

$$
\mathscr{A}(\Phi, \boldsymbol{\zeta})=(Q, \Phi) \quad \forall \Phi \in \mathscr{V} .
$$

It can be observed that if $\Psi$ is an isolated solution of (A.3), then (A.9) and (A.11) are well posed and satisfy the a priori bounds

$$
\|\boldsymbol{\Theta}\|_{2} \leq C\|G\|,\|\boldsymbol{\Theta}\|_{2+\alpha} \leq C\|G\| \text { and }\|\boldsymbol{\zeta}\|_{2} \leq C\|Q\|_{-1},\|\boldsymbol{\zeta}\|_{2+\alpha} \leq C\|Q\|_{-1},
$$

where $\alpha$ is the index of elliptic regularity. The discrete linearized problem is defined as: find $\Theta_{h} \in \mathscr{V}_{h}$ such that

$$
\mathscr{A}_{h}\left(\Theta_{h}, \Phi\right)=(G, \Phi) \quad \forall \Phi \in \mathscr{V}_{h}
$$

where

$$
\mathscr{A}_{h}\left(\Theta_{h}, \Phi\right):=A_{h}\left(\Theta_{h}, \Phi\right)+B_{h}\left(\Psi, \Theta_{h}, \Phi\right)+B_{h}\left(\Theta_{h}, \Psi, \Phi\right)+\mathfrak{C}_{h}\left(\Theta_{h}, \Phi\right) .
$$

With this background, Theorem 3.12, Lemma 4.1 and Theorems 4.2 and 4.8 can be modified for the new formulation, leading to the applicability of the analysis to a more general form of the von Kármán equations. We will sketch the proofs of the important results.

Theorem A.1 (Well posedness of discrete linearized problem). If $\Psi$ is an isolated solution of (A.3), then for sufficiently small $h$, the discrete linearized problem (A.13) is well-posed. 
Outline of the proof. Following the proof of Theorem 3.12, we easily arrive at (3.26) using (A.8). To estimate $\left\|E_{h} \Theta_{h}\right\|_{1}$ in this case, choose $Q=-\Delta E_{h} \Theta_{h}$ and $\Phi=E_{h} \Theta_{h}$ in (A.11) and use (A.13) to obtain

$$
\begin{aligned}
\left\|E_{h} \Theta_{h}\right\|_{1}^{2}= & A_{h}\left(E_{h} \Theta_{h}-\Theta_{h}, \boldsymbol{\zeta}\right)+A_{h}\left(\Theta_{h}, \boldsymbol{\zeta}-\Pi_{h} \boldsymbol{\zeta}\right)+B_{h}\left(\Psi, E_{h} \Theta_{h}, \boldsymbol{\zeta}-\Pi_{h} \boldsymbol{\zeta}\right)+B_{h}\left(E_{h} \Theta_{h}, \Psi, \boldsymbol{\zeta}-\Pi_{h} \boldsymbol{\zeta}\right) \\
& +B_{h}\left(\Psi, E_{h} \Theta_{h}-\Theta_{h}, \Pi_{h} \boldsymbol{\zeta}\right)+B_{h}\left(E_{h} \Theta_{h}-\Theta_{h}, \Psi, \Pi_{h} \boldsymbol{\zeta}\right)+\left(G, \Pi_{h} \boldsymbol{\zeta}\right) \\
& +\left(\mathfrak{C}_{h}\left(E_{h} \Theta-\Theta_{h}, \boldsymbol{\zeta}\right)+\mathfrak{C}_{h}\left(\Theta_{h}, \boldsymbol{\zeta}-\Pi_{h} \boldsymbol{\zeta}\right)\right)
\end{aligned}
$$

The last term can be estimated using (A.8), Lemmas 3.3 and 3.4 as

$$
\left|\mathfrak{C}_{h}\left(E_{h} \Theta-\Theta_{h}, \boldsymbol{\zeta}\right)+\mathfrak{C}_{h}\left(\Theta_{h}, \boldsymbol{\zeta}-\Pi_{h} \boldsymbol{\zeta}\right)\right| \leq C h\|\boldsymbol{\zeta}\|_{2} .
$$

The remaining terms are estimated as in Theorem 3.12 and result follows.

The next lemma follows as in Lemma 4.1 using (A.8) and hence the proof is skipped.

Lemma A.2 (Nonsingularity of perturbed bilinear form). Let $\Pi_{h} \Psi$ be the interpolation of $\Psi$ as defined in Lemma 3.3. Then, for sufficiently small $h$, the perturbed bilinear form defined by

$$
\tilde{\mathscr{A}}_{h}(\Theta, \Phi)=A_{h}(\Theta, \Phi)+B_{h}\left(\Pi_{h} \Psi, \Theta, \Phi\right)+B_{h}\left(\Theta, \Pi_{h} \Psi, \Phi\right)+\mathfrak{C}(\Theta, \Phi)
$$

is nonsingular on $\mathscr{V}_{h} \times \mathscr{V}_{h}$, if (A.14) is nonsingular on $\mathscr{V}_{h} \times \mathscr{V}_{h}$.

Theorem A.3 (Mapping of ball to ball). For a sufficiently small choice of $h$, there exists a positive constant $R(h)$ such that for any $\Theta \in \mathscr{V}_{h}$,

$$
\left\|\Theta-\Pi_{h} \Psi\right\|_{2, h} \leq R(h) \Rightarrow\left\|\mu(\Theta)-\Pi_{h} \Psi\right\|_{2, h} \leq R(h) .
$$

That is, $\mu$ maps the ball $\mathbb{B}_{R(h)}\left(\Pi_{h} \Psi\right)$ to itself.

Outline of the proof. Proceeding as in the proof of Theorem 4.2, using nonsingularity of $\tilde{\mathscr{A}}_{h}(\cdot, \cdot)$ and Lemma A.2, there exists $\bar{\Phi} \in \mathscr{V}_{h}$ such that $\|\bar{\Phi}\|_{2, h}=1$ and

$$
\begin{aligned}
& \frac{\beta}{4}\left\|\mu(\Theta)-\Pi_{h} \Psi\right\|_{2, h} \leq \tilde{\mathscr{A}}_{h}\left(\mu(\Theta)-\Pi_{h} \Psi, \bar{\Phi}\right) \\
& =L_{h}\left(\bar{\Phi}-E_{h} \bar{\Phi}\right)+\left(A_{h}\left(\Psi, E_{h} \bar{\Phi}\right)-A_{h}\left(\Pi_{h} \Psi, \bar{\Phi}\right)\right)+\left(B_{h}\left(\Psi, \Psi, E_{h} \bar{\Phi}\right)-B_{h}\left(\Pi_{h} \Psi, \Pi_{h} \Psi, \bar{\Phi}\right)\right) \\
& \quad+B_{h}\left(\Pi_{h} \Psi-\Theta, \Theta-\Pi_{h} \Psi, \bar{\Phi}\right)+\left(\mathfrak{C}_{h}\left(\Psi, E_{h} \bar{\Phi}\right)-\mathfrak{C}_{h}\left(\Pi_{h} \Psi, \bar{\Phi}\right)\right)=: \sum_{i=1}^{5} T_{i} .
\end{aligned}
$$

The terms $T_{1}$ to $T_{4}$ can be estimated as in the proof of Theorem 4.2. The last term $T_{5}$ is estimated using (A.8), Lemmas 3.3 and 3.4 as:

$$
\left|\mathfrak{C}_{h}\left(\Psi, E_{h} \bar{\Phi}\right)-\mathfrak{C}_{h}\left(\Pi_{h} \Psi, \bar{\Phi}\right)\right| \leq\left|\mathfrak{C}_{h}\left(\Psi, E_{h} \bar{\Phi}-\bar{\Phi}\right)\right|+\left|\mathfrak{C}_{h}\left(\Psi-\Pi_{h} \Psi, \bar{\Phi}\right)\right| \leq C h\|\Psi\|_{2} .
$$

The remaining proof follows exactly same as the proof of Theorem 4.2.

The existence of solution $\Psi_{h}$ of (A.5) follows using Theorem A.3 and satisfies the estimate

$$
\left\|\Psi_{h}-\Pi_{h} \Psi\right\|_{2, h} \leq C h^{\alpha} .
$$

A contraction result similar to Theorem 4.4 also holds true in this case. The energy estimate follows exactly as in the proof of Theorem 4.6. 
Theorem A.4 ( $H^{1}$ estimate). Let $\Psi$ and $\Psi_{h}$ be the solutions of (A.3) and (A.5) respectively. Assume that $\Psi$ is an isolated solution. Then, for sufficiently small h, it holds

$$
\left\|\Psi-\Psi_{h}\right\|_{1, h} \leq C h^{2 \alpha}
$$

where $\alpha \in\left(\frac{1}{2}, 1\right]$ is the index of elliptic regularity.

Outline of the proof. A use of triangle inequality yields

$$
\left\|\Psi-\Psi_{h}\right\|_{1, h} \leq\left\|\Psi-\Pi_{h} \Psi\right\|_{1, h}+\left\|\Pi_{h} \Psi-\Psi_{h}\right\|_{1, h} \leq\left\|\Psi-\Pi_{h} \Psi\right\|_{1, h}+\left\|\boldsymbol{\rho}-E_{h} \rho\right\|_{1, h}+\left\|E_{h} \boldsymbol{\rho}\right\|_{1},
$$

where $\boldsymbol{\rho}=\Pi_{h} \Psi-\Psi_{h}$. A choice of $Q=-\Delta E_{h} \boldsymbol{\rho}$ and $\Phi=E_{h} \boldsymbol{\rho}$ in the dual problem (A.11) leads to

$$
\begin{aligned}
\left(\nabla E_{h} \boldsymbol{\rho}, \nabla E_{h} \boldsymbol{\rho}\right)= & \mathscr{A}_{h}\left(E_{h} \boldsymbol{\rho}, \boldsymbol{\zeta}\right)=\mathscr{A}_{h}\left(E_{h} \boldsymbol{\rho}-\boldsymbol{\rho}, \boldsymbol{\zeta}\right)+\mathscr{A}_{h}(\boldsymbol{\rho}, \boldsymbol{\zeta}) \\
= & A_{h}\left(E_{h} \boldsymbol{\rho}-\boldsymbol{\rho}, \boldsymbol{\zeta}\right)+B_{h}\left(\Psi, E_{h} \boldsymbol{\rho}-\boldsymbol{\rho}, \boldsymbol{\zeta}\right)+B_{h}\left(E_{h} \boldsymbol{\rho}-\boldsymbol{\rho}, \Psi, \boldsymbol{\zeta}\right)+\mathfrak{C}_{h}\left(E_{h} \boldsymbol{\rho}-\boldsymbol{\rho}, \boldsymbol{\zeta}\right) \\
& +A_{h}\left(\Pi_{h} \Psi-\Psi, \boldsymbol{\zeta}\right)+A_{h}\left(\Psi-\Psi_{h}, \boldsymbol{\zeta}-\Pi_{h} \boldsymbol{\zeta}\right)+A_{h}\left(\Psi, \Pi_{h} \boldsymbol{\zeta}-\boldsymbol{\zeta}\right)+L_{h}\left(\boldsymbol{\zeta}-\Pi_{h} \boldsymbol{\zeta}\right) \\
& +\left(B_{h}\left(\Psi, \Pi_{h} \Psi-\Psi_{h}, \boldsymbol{\zeta}\right)+B_{h}\left(\Pi_{h} \Psi-\Psi_{h}, \Psi, \boldsymbol{\zeta}\right)-B_{h}(\Psi, \Psi, \boldsymbol{\zeta})+B_{h}\left(\Psi_{h}, \Psi_{h}, \Pi_{h} \boldsymbol{\zeta}\right)\right) \\
& +\left(\mathfrak{C}_{h}\left(\Pi_{h} \Psi-\Psi, \boldsymbol{\zeta}\right)+\mathfrak{C}_{h}\left(\Psi_{h}, \Pi_{h} \boldsymbol{\zeta}-\boldsymbol{\zeta}\right)\right)
\end{aligned}
$$

Combining all the terms related to $\mathfrak{C}_{h}$ and using (A.8), (A.18) and Lemmas 3.3, 3.4, we obtain the estimate

$$
\mathfrak{C}_{h}\left(E_{h} \boldsymbol{\rho}-\boldsymbol{\rho}, \boldsymbol{\zeta}\right)+\mathfrak{C}_{h}\left(\Pi_{h} \Psi-\Psi, \boldsymbol{\zeta}\right)+\mathfrak{C}_{h}\left(\Psi_{h}, \Pi_{h} \boldsymbol{\zeta}-\boldsymbol{\zeta}\right) \leq C h^{1+\alpha}\|\boldsymbol{\zeta}\|_{2+\alpha} .
$$

Estimating the remaining terms as in the proof of Theorem 4.7, the result follows.

The Newton's iterates in this case are defined by

$$
A_{h}\left(\Psi_{h}^{n}, \Phi\right)+B_{h}\left(\Psi_{h}^{n-1}, \Psi_{h}^{n}, \Phi\right)+B_{h}\left(\Psi_{h}^{n}, \Psi_{h}^{n-1}, \Phi\right)+\mathfrak{C}_{h}\left(\Psi_{h}^{n}, \Phi\right)=B_{h}\left(\Psi_{h}^{n-1}, \Psi_{h}^{n-1}, \Phi\right)+L_{h}(\Phi) \quad \forall \Phi \in \mathscr{V}_{h} .
$$

The quadratic convergence result follows by a similar proof as in Theorem 4.8.

Example A.5. In this example, we perform numerical experiments for the problem (A.1), (A.2) with $p / D=10$, over a unit square domain. Choose the right hand side load functions such that the exact solution is given by

$$
u(x, y)=x^{2}(1-x)^{2} y^{2}(1-y)^{2}, \quad v(x, y)=\sin ^{2}(\pi x) \sin ^{2}(\pi y) .
$$

We consider the same initial triangulation and its uniform refinement process as in Example 5.1. Tables A.1 and A.2 show the errors and experimental convergence rates for the variables $u_{h}$ and $v_{h}$. The computational order of convergences in broken $H^{2}, H^{1}$ norms are quasi-optimal and verify the theoretical results. Also, the order of convergence with respect to $L^{2}$ norm is sub-optimal justifying the results in [17].

TABle A.1. Errors and Convergence rates of $u_{h}$ in broken $H^{2}, H^{1}$ and $L^{2}$ norms.

\begin{tabular}{lcccccc}
\hline \# unknowns & $\left|u-u_{h}\right|_{2, h}$ & Order & $\left|u-u_{h}\right|_{1, h}$ & Order & $\left\|u-u_{h}\right\|_{L^{2}}$ & Order \\
\hline 25 & $0.101724 \mathrm{E}-0$ & - & $0.129574 \mathrm{E}-1$ & - & $0.469669 \mathrm{E}-2$ & - \\
113 & $0.391714 \mathrm{E}-1$ & 1.3767 & $0.275863 \mathrm{E}-2$ & 2.2317 & $0.957470 \mathrm{E}-3$ & 2.2943 \\
481 & $0.195023 \mathrm{E}-1$ & 1.0061 & $0.767382 \mathrm{E}-3$ & 1.8459 & $0.252196 \mathrm{E}-3$ & 1.9246 \\
1985 & $0.974844 \mathrm{E}-2$ & 1.0004 & $0.198544 \mathrm{E}-3$ & 1.9504 & $0.641987 \mathrm{E}-4$ & 1.9739 \\
8065 & $0.487399 \mathrm{E}-2$ & 1.0000 & $0.500990 \mathrm{E}-4$ & 1.9866 & $0.161298 \mathrm{E}-4$ & 1.9928 \\
32513 & $0.243697 \mathrm{E}-2$ & 1.0000 & $0.125546 \mathrm{E}-4$ & 1.9965 & $0.403763 \mathrm{E}-5$ & 1.9981 \\
\hline
\end{tabular}


TABLE A.2. Errors and Convergence rates of $v_{h}$ in broken $H^{2}, H^{1}$ and $L^{2}$ norms.

\begin{tabular}{ccccccc}
\hline \# unknowns & $\left|v-v_{h}\right|_{2, h}$ & Order & $\left|v-v_{h}\right| 1, h$ & Order & $\left\|v-v_{h}\right\|_{L^{2}}$ & Order \\
\hline 25 & 19.245650 & - & $2.140609 \mathrm{E}-0$ & - & $0.770875 \mathrm{E}-0$ & - \\
113 & 9.5043692 & 1.0178 & $0.569978 \mathrm{E}-0$ & 1.9090 & $0.177898 \mathrm{E}-0$ & 2.1154 \\
481 & 5.0549208 & 0.9109 & $0.161737 \mathrm{E}-0$ & 1.8172 & $0.482777 \mathrm{E}-1$ & 1.8816 \\
1985 & 2.5758938 & 0.9726 & $0.421546 \mathrm{E}-1$ & 1.9398 & $0.123930 \mathrm{E}-1$ & 1.9618 \\
8065 & 1.2944929 & 0.9926 & $0.106618 \mathrm{E}-1$ & 1.9832 & $0.312076 \mathrm{E}-2$ & 1.9895 \\
32513 & 0.6480848 & 0.9981 & $0.267351 \mathrm{E}-2$ & 1.9956 & $0.781643 \mathrm{E}-3$ & 1.9973 \\
\hline
\end{tabular}

Acknowledgements. The authors would like to sincerely thank Professors S.C. Brenner and Li-yeng Sung for their suggestions on extension of the results to non-convex polygonal domains and to Dr. Thirupathi Gudi for his comments. The first author would also like to thank National Board for Higher Mathematics (NBHM) for the financial support towards the research work.

\section{REFERENCES}

[1] J. Alberty, C. Carstensen and S.A. Funken, Remarks around 50 lines of Matlab: short finite element implementation. Numer. Algorithms 20 (1999) 117-137.

[2] M.S. Berger, On von Kármán equations and the buckling of a thin elastic plate, I the clamped plate. Commun. Pure Appl. Math. 20 (1967) 687-719.

[3] M.S. Berger, Nonlinearity and functional analysis. Academic Press (1977).

[4] M.S. Berger and P.C. Fife, On von Kármán equations and the buckling of a thin elastic plate. Bull. Amer. Math. Soc. 72 (1966) 1006-1011.

[5] M.S. Berger and P.C. Fife, Von Kármán equations and the buckling of a thin elastic plate. II plate with general edge conditions. Commun. Pure Appl. Math. 21 (1968) 227-241.

[6] H. Blum and R. Rannacher, On the boundary value problem of the biharmonic operator on domains with angular corners. Math. Methods Appl. Sci. 2 (1980) 556-581.

[7] D. Braess, Finite Elements, Theory, Fast Solvers, and Applications in Elasticity Theory, 3rd edition. Cambridge (2007).

[8] S.C. Brenner, Forty years of the Crouzeix-Raviart element. Numer. Methods Partial Differ. Equations 31 (2015) $367-396$.

[9] S.C. Brenner, T. Gudi, M. Neilan and L.-Y. Sung, $C^{0}$ penalty methods for the fully nonlinear Monge-Ampère equation. Math. Comput. 80 (2011) 1979-1995.

[10] S.C. Brenner and L. R. Scott, The mathematical theory of finite element methods, 3rd edition. Springer (2007).

[11] S. C. Brenner, L.-Y. Sung, H. Zhang and Y. Zhang, A Morley finite element method for the displacement obstacle problem of clamped Kirchhoff plates. J. Comput. Appl. Math. 254 (2013) 31-42.

[12] F. Brezzi, Finite element approximations of the von Kármán equations. RAIRO Anal. Numér. 12 (1978) 303-312.

[13] P.G. Ciarlet, The finite element method for elliptic problems. North-Holland, Amsterdam (1978).

[14] P. G. Ciarlet, Mathematical Elasticity: Theory of Plates. In vol. II. North-Holland, Amsterdam (1997).

[15] L.C. Evans, Partial Differential Equations. In vol. 19. American Mathematical Society (1998).

[16] P. Grisvard, Singularities in Boundary Value Problems. Vol. RMA 22. Masson \& Springer-Verlag (1992).

[17] J. Hu and Z.C. Shi, The best $L^{2}$ norm error estimate of lower order finite element methods for the fourth order problem. $J$. Comput. Math. 30 (2012) 449-460.

[18] S. Kesavan, Topics in Functional Analysis and Applications. New Age International Publishers (2008).

[19] G. H. Knightly, An existence theorem for the von Kármán equations. Arch. Ration. Mech. Anal. 27 (1967) $233-242$.

[20] P. Lascaux and P. Lesaint, Some nonconforming finite elements for the plate bending problem. RAIRO Anal. Numér. 9 (1975) 9-53.

[21] W. Ming and J. Xu, The Morley element for fourth order elliptic equations in any dimensions. Numer. Math. 103 (2006) $155-169$.

[22] T. Miyoshi, A mixed finite element method for the solution of the von Kármán equations. Numer. Math. 26 (1976) 255-269.

[23] M. Neilan, A nonconforming Morley finite element method for the fully nonlinear Monge-Ampère equation. Numer. Math. 115 (2010) 371-394.

[24] A. Quarteroni, Hybrid finite element methods for the von Kármán equations. Calcolo 16 (1979) 271-288.

[25] L. Reinhart, On the numerical analysis of the von Kármán equations: mixed finite element approximation and continuation techniques. Numer. Math. 39 (1982) 371-404.

[26] X. Xu, S.H. Lui and T. Rahaman, A two level additive Schwarz method for the Morley nonconforming element approximation of a nonlinear biharmonic equation. IMA J. Numer. Anal. 24 (2004) 97-122. 